\title{
TIME-PERIODIC LINEARIZED SOLUTIONS OF THE COMPRESSIBLE EULER EQUATIONS AND A PROBLEM OF SMALL DIVISORS*
}

\author{
BLAKE TEMPLE ${ }^{\dagger}$ AND ROBIN YOUNG
}

\begin{abstract}
It has been unknown since the time of Euler whether or not time-periodic sound wave propagation is physically possible in the compressible Euler equations, due mainly to the ubiquitous formation of shock waves. The existence of such waves would confirm the possibility of dissipation free long distance signaling. Following our work in [B. Temple and R. Young, $A$ paradigm for time-periodic sound wave propagation in the compressible Euler equations, Methods Appl. Anal., 16 (2009), pp. 341-363], we derive exact linearized solutions that exhibit the simplest possible periodic wave structure that can balance compression and rarefaction along characteristics in the nonlinear Euler problem. These linearized waves exhibit interesting phase and group velocities analogous to linear dispersive waves. Moreover, when the spatial period is incommensurate with the time period, the sound speed is incommensurate with the period, and a new periodic wave pattern is observed in which the sound waves move in a quasiperiodic trajectory though a periodic configuration of states. This establishes a new way in which nonlinear solutions that exist arbitrarily close to these linearized solutions can balance compression and rarefaction along characteristics in a quasiperiodic sense. We then rigorously establish the spectral properties of the linearized operators associated with these linearized solutions. In particular we show that the linearized operators are invertible on the complement of a one-dimensional kernel containing the periodic solutions only in the case when the wave speeds are incommensurate with the periods, but these invertible operators have small divisors, analogous to KAM theory. Almost everywhere algebraic decay rates for the small divisors are proven. In particular this provides a solid framework for the problem of perturbing these linearized solutions to exact nonlinear periodic solutions of the fully compressible Euler equations.
\end{abstract}

Key words. compressible Euler, periodic solutions, small divisors

AMS subject classifications. 35L65, 76N10, 35Q31

DOI. $10.1137 / 080739604$

1. Introduction. Time-periodic solutions of the compressible Euler equations represent dissipation free long distance signaling at the level of sound waves, but it has been unknown since the time of Euler whether or not there exist time-periodic solutions that satisfy the compressible Euler equations exactly. Since Riemann demonstrated that shock waves form in solutions, experts have believed that time-periodic solutions of the compressible Euler equations with sound wave propagation were physically impossible due to the belief that shock waves could not be ruled out in oscillatory solutions: shock waves introduce increase of entropy and dissipation inconsistent with time-periodic evolution [11]. This belief was supported in 1970 by the definitive paper of Glimm and Lax [6], which proved that for the reduced Euler equations corresponding to isentropic flow, solutions starting from space-periodic initial data must form shock waves and decay (by shock wave dissipation) to the constant state average in each period, at a rate $1 / t$. Subsequent work kindled the idea that periodic wave propa-

*Received by the editors October 27, 2008; accepted for publication (in revised form) September 8, 2010; published electronically January 4, 2011.

http://www.siam.org/journals/sima/43-1/73960.html

${ }^{\dagger}$ Department of Mathematics, University of California, Davis, CA 95616 (temple@math.ucdavis. edu). This author's research was supported in part by NSF Applied Mathematics grant DMS-0406096.

${ }^{\ddagger}$ Department of Mathematics and Statistics, University of Massachusetts, Amherst, MA 01003 (young@math.umass.edu). This author's research was supported in part by NSF Applied Mathematics grant DMS-050-7884. 
gation is possible when the entropy fields are nonconstant [18, 19, 20, 22, 28, 24, 31, 33]. Numerical simulations by Shefter and Rosales [22] and Vaynblat [28] indicated that solutions evolving through nonconstant space-periodic entropy fields do not decay to the constant state average in each period, but rather appear to evolve into periodic or quasiperiodic evolution. Within this context, the authors in [26] derived the simplest possible periodic wave structure ${ }^{1}$ consistent with time-periodic sound wave propagation in the $3 \times 3$ nonlinear compressible Euler equations, a wave structure that requires at least three coupled nonlinear equations to support it. This simplest wave pattern was derived by combinatorial considerations based on a classification of compressive and rarefactive wave interactions at entropy jumps, using the starting principle that shock free periodic or quasiperiodic solutions of compressible Euler should balance compression and rarefaction along every characteristic (sound wave).

In this paper we construct exact linearized solutions of Euler that exhibit the simplest periodic wave structure identified in [26] for the nonlinear problem. For this we start by constructing a nonlinear eigenvalue problem whose solutions correspond to nonlinear periodic solutions of the compressible Euler equations having the simplest structure identified in [26]. The nonlinear operator involved encodes evolution in space starting from time-periodic data posed at an entropy jump. (In Lagrangian coordinates, entropy jumps propagate at zero speed.) Trivial solutions of this eigenvalue problem correspond to periodic solutions of the compressible Euler equations consisting of piecewise constant states separated by two entropy jumps. Linearizing around this solution we obtain a linearized eigenvalue problem whose solutions we expect will perturb to solutions of the nonlinear problem because they encode the structure identified in [26]. This linearized operator is nonsymmetric, and consists of the composition of five elementary linear operators that do not commute: a linear evolution at the first entropy level, followed by an entropy jump, followed by linear evolution at the second entropy level, followed by the inverse entropy jump, followed by a half period shift. The combination of shifts and jumps ensures the mixing of compression and rarefaction along characteristics under nonlinear perturbation, and highly restricts the kernel of the linearized operator. We next derive a condition relating the magnitude of the entropy jump to the period that guarantees the existence of a solution to the linear eigenvalue problem in the Fourier 1-mode. We then obtain our linearized periodic solutions by deriving closed form expressions for the resulting 1-mode solutions of the linear eigenvalue problem. To get closed form expressions for solutions, we introduce a new nondimensional form of the Euler equations for which linearized evolution is represented by rotation in the complex plane.

The resulting linearized solutions display, in closed form expression, the propagation properties of nearby nonlinear sound waves that formally balance compression and rarefaction along characteristics. In this sense, the solutions exhibit the simplest possible mechanisms for dissipation free transmission of sound waves in the nonlinear problem. In the simplest case, each characteristic sound wave traverses eight entropy levels before periodic return, and the wave crests propagate at a speed different from a shock or sound speed; cf. [26]. But the linearized solutions constructed here show that more complicated ways of balancing compression and rarefaction are possible due

\footnotetext{
${ }^{1}$ We say that a periodic or quasiperiodic wave structure is possible, at a formal level, if each characteristic (sound wave) traverses both regions of compression and rarefaction, and that these are formally in balance. Whether such a possible formal wave structure actually exists in a true periodic solution of Euler is then a deep mathematical question as to whether the data can be tuned to bring compression and rarefaction precisely into balance, so that shock wave formation is prevented.
} 
to the fact that it is consistent at the linearized level for solutions to move through periods at speeds that are incommensurate with the speed of the period. In this case, the solution produces a periodic tiling of the $x t$-plane that is periodic in space and time, but sound waves traverse the periodic tiling in a quasiperiodic fashion. Even so, compression and rarefaction will be in balance formally in nearby nonlinear solutions because of the ergodicity of the quasiperiodic motion of the sound wave relative to the tiling.

The structure of the linearized waves carries over approximately to nearby nonlinear solutions, but a mathematical proof is needed to demonstrate rigorously that solutions of the linear eigenvalue problem carry over exactly to solutions of the nonlinear eigenvalue problem. Motivated by this perturbation problem, in section 6 we analyze the spectrum of the linearized operator that expresses the eigenvalue problem corresponding to periodicity. We prove that our exact periodic solutions correspond to eigenvectors in the 1-mode kernel of the associated linearized operators, and that for almost every choice of periods, the linearized operator is invertible on the complement of the kernel. Interestingly, the linearized solutions can be isolated in the kernel of the linearized operator only in the case when the sound speeds are incommensurate with the periods. We end the section with a proof, based on Liouville numbers, that for special choices of periods, the eigenvalues are bounded away from zero by algebraic rates. Numerical results are also presented, showing that the eigenmodes with small divisors can be very sparse, for example, nicely bounded away from zero in the first fifty modes.

This analysis of linearized solutions and corresponding linearized operators casts the perturbation problem for the existence of time-periodic solutions of compressible Euler into a form amenable to a Lyapunov-Schmidt decomposition. That is, to obtain periodic solutions of a nonlinear problem by perturbation from a known solution of a linearized problem by bifurcation methods, a major step is to show that the linearized operator is invertible on the orthogonal complement of the known solution; cf. [7]. This we accomplish in section 7 . Once this is accomplished, the central issue in the completion of a rigorous proof that periodic solutions exist (i.e., that linearized solutions perturb to nearby nonlinear solutions) is then the problem of establishing an implicit function theorem based on the resulting invertible linearized operator (the so-called auxiliary equation [7]). The proof of such an implicit function theorem when small divisors are present is then problematic (see, e.g., [2]). For us, the small divisors are estimated by algebraic decay rates, and the resulting implicit function theorem is analogous to problems in KAM theory [4, 1], but for us a proof must face the small divisor issue in a quasilinear problem, a setting for which the current mathematical technology does not directly apply; cf. [2]. The generation of such a proof is the topic of the authors' ongoing research program. In the follow-up paper [27], the authors show that the Lyapunov-Schmidt decomposition is consistent, and that periodic solutions exist subject to an arbitrarily large Fourier mode cutoff. Given the successes of KAM theory, one can expect only that the technical obstacle of the small divisors will be overcome and these solutions perturb to exact periodic solutions of the compressible Euler equations. The methods here also provide a starting point for the numerical simulation of time-periodic sound waves.

\section{Background.}

2.1. The compressible Euler equations. The compressible Euler equations describe the time evolution of a perfect fluid in the absence of dissipative effects. 
These are

$$
\begin{aligned}
\rho_{t}+\operatorname{div}[\rho \mathbf{u}] & =0, \\
\left(\rho u^{i}\right)_{t}+\operatorname{div}\left[\rho u^{i} \mathbf{u}\right] & =-\nabla p, \\
E_{t}+\operatorname{div}[(E+p) \mathbf{u}] & =0
\end{aligned}
$$

describing the evolution of the density $\rho$, velocity $\mathbf{u} \in \mathbb{R}^{3}$, and energy density $E=$ $\frac{1}{2} \rho \mathbf{u}^{2}+\rho \varepsilon$, where $\varepsilon$ is the specific internal energy. To close the system, an equation of state is given which relates the pressure $p$ to $\varepsilon$ and $\rho$. We consider a polytropic gamma-law gas, described by

$$
\varepsilon=c_{\tau} \tau^{-(\gamma-1)} e^{S / c_{\tau}} \quad \text { and } \quad p=\frac{c_{\tau}}{\gamma-1} \tau^{-\gamma} e^{S / c_{\tau}}
$$

where $\tau=1 / \rho$ is the specific volume, $S$ is the specific entropy, $\gamma>1$ is the adiabatic gas constant, and $c_{\tau}$ the specific heat [23]. For smooth solutions, the energy equation (3) is equivalent to the adiabatic constraint or entropy equation

$$
(\rho S)_{t}+\operatorname{div}(\rho S \mathbf{u})=0,
$$

which states that entropy is transported with the fluid [23].

For sound wave propagation in one direction $x^{\prime}$, the equations reduce to the system of $3 \times 3$ Euler equations

$$
\begin{aligned}
\rho_{t}+(\rho u)_{x^{\prime}} & =0 \\
(\rho u)_{t}+\left(\rho u^{2}+p\right)_{x^{\prime}} & =0 \\
E_{t}+[(E+p) u]_{x^{\prime}} & =0
\end{aligned}
$$

In a Lagrangian frame of reference, which moves with the fluid, the equations can be written

$$
\begin{aligned}
\tau_{t}-u_{x} & =0, \\
u_{t}+p_{x} & =0, \\
E_{t}^{*}+(u p)_{x} & =0,
\end{aligned}
$$

where now $x$ represents the material coordinate for the fluid, given by

$$
x=\int \rho d x^{\prime}
$$

where $x^{\prime}$ is the spatial coordinate, and $E^{*}=E / \rho=\frac{1}{2} u^{2}+\varepsilon$. In this Lagrangian frame, the adiabatic constraint (5) takes on the particularly simple form

$$
S_{t}=0
$$

which can be used instead of (5) on smooth solutions [23].

Because we are considering solutions which are (piecewise) smooth, it is enough to consider (6), (7), and (9). We recall from our previous paper [26] the convenient change of variables

$$
m=e^{S / 2 c_{\tau}} \quad \text { and } \quad z=K_{z} \tau^{-\frac{\gamma-1}{2}},
$$


so that (4) becomes

$$
\varepsilon=K_{\varepsilon} m^{2} z^{2} \quad \text { and } \quad p=K_{p} m^{2} z^{\frac{2 \gamma}{\gamma-1}}
$$

where $K$ 's are appropriately given constants. In these variables, for smooth solutions, (6)-(9) can be written as the quasilinear system

$$
\begin{gathered}
z_{t}+\frac{c}{m} u_{x}=0, \\
u_{t}+m c z_{x}+2 \frac{p}{m} m_{x}=0, \\
m_{t}=0
\end{gathered}
$$

where we have used (9) in place of (8); here $c$ is the Lagrangian sound speed, defined by

$$
c(\tau, S)=\sqrt{-p_{\tau}(\tau, S)}
$$

which becomes

$$
c(m, z)=K_{c} m z^{\frac{\gamma+1}{\gamma-1}}
$$

Recall that a strictly hyperbolic $3 \times 3$ system has three wave families, each corresponding to an eigenvalue or wavespeed of the system. In the Lagrangian frame, the wavespeeds of system (11) are $\pm c$ and 0 . The nonlinear waves are the forward $(+$ or 3 -wave, speed $+c)$ and backward ( - or 1-wave, speed $-c$ ) waves, along which sound waves propagate. The stationary ( 0 or 2 -wave, speed 0 ) waves are contact or jump discontinuities which propagate with the fluid and which are linearly degenerate. Our forward and backward waves are simple waves which are either rarefactive or compressive; we do not treat shocks here as they are incompatible with periodic wave propagation.

We consider solutions in which the entropy $m$ (or $S$ ) is piecewise constant, varying periodically in space but stationary in time. To resolve the jump in variables across the entropy jumps, we must apply the Rankine-Hugoniot relations for (6)-(8), which are

$$
\begin{aligned}
{[u] } & =s[-\tau], \\
{[p] } & =s[u], \\
{[u p] } & =s\left[\frac{1}{2} u^{2}+\varepsilon\right],
\end{aligned}
$$

where, as usual, $s$ is the speed of propagation of the discontinuity and [.] is the jump. Since we are concerned only with contact discontinuities, we take $s=0$, and the jump conditions become $[u]=[p]=0$, or

$$
u_{L}=u_{R} \quad \text { and } \quad m_{L} z_{L}^{\frac{\gamma-1}{\gamma}}=m_{R} z_{R}^{\frac{\gamma-1}{\gamma}}
$$

On regions where the entropy is constant, the $3 \times 3$ system (11) reduces to the $2 \times 2$ quasilinear system

$$
\begin{aligned}
z_{t}+\frac{c}{m} u_{x} & =0, \\
u_{t}+m c z_{x} & =0
\end{aligned}
$$

which is just the $p$-system using $z$ as the thermodynamic coordinate [23]. 
2.2. Wave interactions and character change. In [26], we described the mechanism of wave interactions which can prevent shock formation and, consequently, support nondecaying periodic solutions. The key observation is to note that although a simple wave preserves its rarefactive or compressive character (before shock formation) when the entropy is constant, this character can change as the wave crosses a stationary jump discontinuity. Physically, if a simple wave crosses a jump, a simple wave of the opposite family is reflected. If we (nonlinearly) superimpose two such interactions, then one of the reflected waves could be larger than the corresponding transmitted wave, resulting in the outgoing wave having a different character from the corresponding incident wave. We then obtained the periodic structure by carefully combining many such interactions into a consistent wave pattern [26].

To describe the simple waves, we fix the entropy $m$ and write system (15) in the Riemann invariant form

$$
\begin{aligned}
& r_{t}-c r_{x}=0, \\
& s_{t}+c s_{x}=0,
\end{aligned}
$$

where the Riemann invariants are

$$
r=u-m z \quad \text { and } \quad s=u+m z,
$$

respectively. It is well known that for a $2 \times 2$ system, the simple waves are described by the constancy of Riemann invariants [11,23]. That is, the backward simple waves are described by $s=$ const, while the forward waves are given by $r=$ const. Also (16) states that $r$ and $s$ are constant along backward and forward characteristics, respectively, which are the straight lines given by

$$
\frac{d x}{d t}=-c \quad \text { and } \quad \frac{d x}{d t}=c,
$$

respectively.

We summarize by describing both forward and backward simple waves by the equation

$$
u_{R}-u_{L}=m\left(z_{A}-z_{B}\right)
$$

where the subscripts refer to the states to the right, to the left, ahead, and behind the wave, respectively; recall that the entropy $m$ is constant in the wave.

The character of a simple wave is rarefactive $(\mathrm{R})$ or compressive $(\mathrm{C})$ according to whether the sound speed $c$ increases or decreases from ahead (A) to behind (B) the wave, respectively, that is, if the speed ahead is larger, then the wave is rarefactive. Since $d c / d z>0$ and $z=\frac{s-r}{2 m}$, we can also find the character of a wave by checking the time derivative of the appropriate Riemann invariant: for example, if $r_{t} \leq 0$, the backward wave (on which $s$ is constant) is compressive. We thus have the following characterization of simple waves: the wave is

$$
\begin{array}{rll}
\text { Forward } R & \text { iff } & s_{t} \leq 0, \\
\text { Forward } C & \text { iff } & s_{t} \geq 0, \\
\text { Backward } R & \text { iff } & r_{t} \geq 0, \\
\text { Backward } C & \text { iff } & r_{t} \leq 0 .
\end{array}
$$


It is shown in [26] that the $R / C$ character of a simple wave is unchanged as long as the entropy remains constant; however, at an entropy jump, the $R / C$-character can change, as follows. By (14), $u$ is constant across the jump, but $m z$ changes by the scalar factor $q_{L}^{R} \equiv\left(m_{R} / m_{L}\right)^{\frac{1}{\gamma}}$. Thus the Riemann invariants and their time derivatives change, and if $r_{t}$ or $s_{t}$ changes sign, the corresponding wave changes character. We have the following lemma, which is proved in detail in [26].

LEммa 1. The following inequalities characterize when a nonlinear wave changes its $R / C$ value at an entropy jump:

$$
\begin{aligned}
& R_{\text {in }}^{-} \rightarrow C_{\text {out }}^{-} \quad \text { iff } \quad q_{L}^{R} m_{L} \dot{z}_{L}<\dot{u}_{L}<m_{L} \dot{z}_{L}, \\
& C_{\text {in }}^{-} \rightarrow R_{\text {out }}^{-} \quad \text { iff } \quad m_{L} \dot{z}_{L}<\dot{u}_{L}<q_{L}^{R} m_{L} \dot{z}_{L}, \\
& R_{\text {in }}^{+} \rightarrow C_{\text {out }}^{+} \quad \text { iff } \quad-q_{L}^{R} m_{L} \dot{z}_{L}<\dot{u}_{L}<-m_{L} \dot{z}_{L}, \\
& C_{\text {in }}^{+} \rightarrow R_{\text {out }}^{+} \quad \text { iff } \quad-m_{L} \dot{z}_{L}<\dot{u}_{L}<-q_{L}^{R} m_{L} \dot{z}_{L},
\end{aligned}
$$

where $q_{L}^{R}=\left(m_{R} / m_{L}\right)^{\frac{1}{\gamma}}$ is the scalar by which the quantity $m z$ jumps.

2.3. Periodic structure of solutions. We note that the inequalities of Lemma 1 are exclusive, which means that at most one wave can change its character at any time. Our main goal in [26] was to exploit this local change in a single simple wave to build a global wave pattern in which each compression wave changes character before it collapses into a shock wave. By carefully considering the consistency of the wave pattern as built up from many interactions satisfying the lemma, we constructed the simplest possible periodic wave pattern in which compression and rarefaction is balanced along each characteristic. By this we mean that as each forward or backward characteristic is traced through the solution, it passes through stages of compression and rarefaction, and on average the amount of compression and rarefaction are balanced. In this way each compressive characteristic in the solution changes to rarefaction before it collapses into a shock. Since there are no shocks, the solution is time-reversible, and rarefactions are essentially equivalent to compressions.

Our construction of a global periodic wave pattern can be represented as the formal characteristic $x t$-space diagram shown in Figure 1. In this diagram, the vertical lines represent stationary jump discontinuities at which the (piecewise constant) entropy changes: there are two entropy levels corresponding to the narrow and wide strips, respectively. We have represented the forward and backward characteristics as straight lines, with the differing slopes indicating changing wavespeed, and have shaded one complete forward wave consisting of four rarefactive pieces followed by four compressive pieces. The thick lines correspond to "max/min" characteristics, along which the extremal values of the Riemann invariants propagate, and which mark the boundaries between regions of rarefaction and compression. Note that at each entropy jump, part of each wave changes its character.

Figure 1 is a "cartoon" of a periodic solution to the nonlinear Euler equations in that it does not correspond to an actual exact solution. We can, however, make the figure increasingly accurate by considering smaller and smaller amplitudes for the waves, so that the characteristics become closer and closer to parallel straight lines as the wavespeed $c(m, z) \rightarrow c_{0}$. Indeed, in the limit of small amplitudes, all characteristics would have constant speeds, although these would be different at the different entropy levels.

In Figure 2 we show a refined picture of the simplest periodic structure in the limit of small amplitudes, with one tile of the $(x, t)$-plane shaded. We have labeled the 


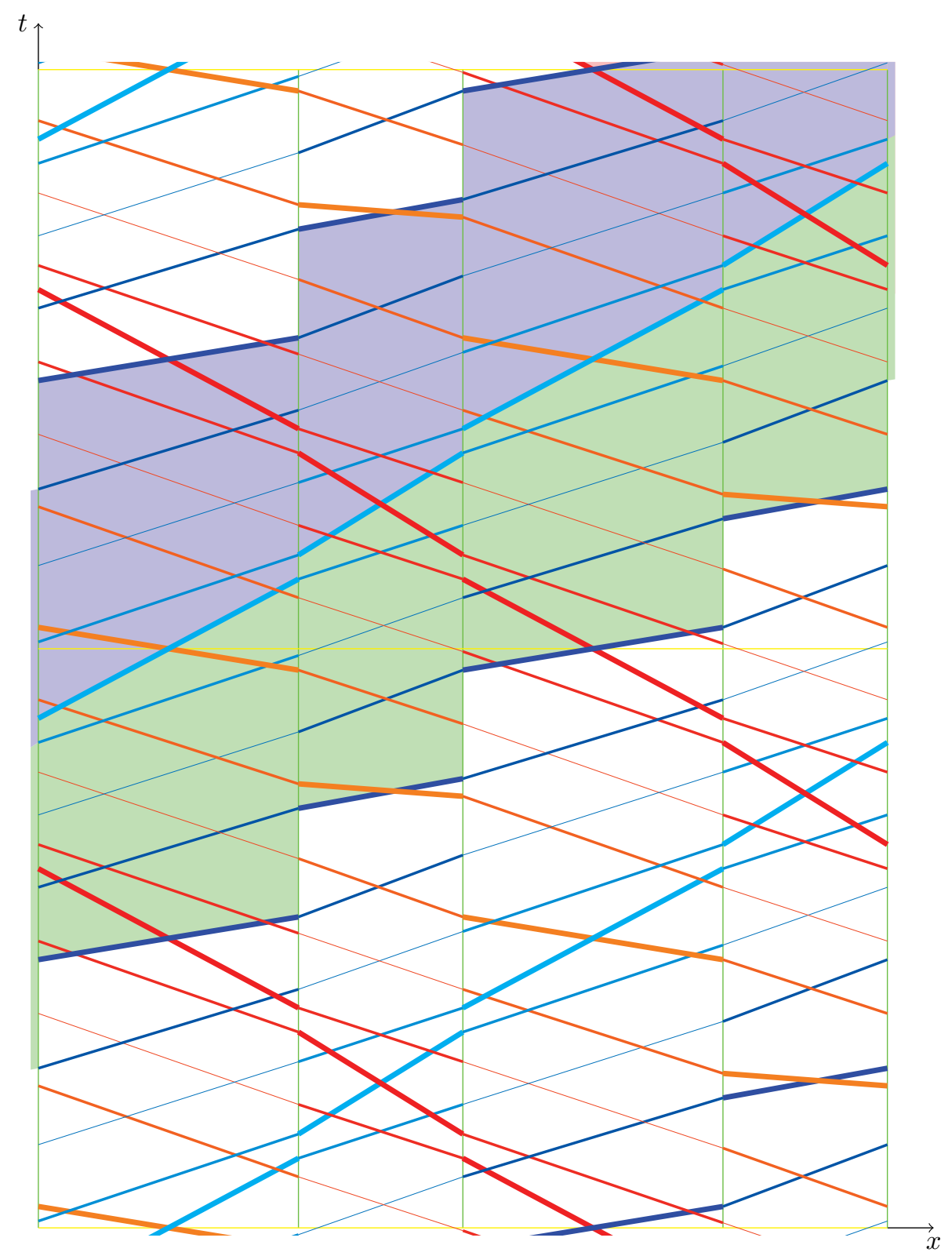

FIG. 1. A periodic pattern incorporating compression and rarefaction.

states in the $(x, t)$-plane as follows: numbers and letters represent states having the same Riemann invariant values $s$ and $r$ on a single entropy level, respectively; these code the relative position of the Riemann invariant inside the wave. Thus, $a$ and $e$ refer to the minimum and maximum $r$ values, and $b, c$, and $d$ are always part of the backward "rarefaction" wave, corresponding to $r_{t} \geq 0$. The states on either side of each entropy jump are also labeled with $\left.{ }^{\sim}\right|^{\sim}$ and ${ }^{\wedge} \mid$; , respectively. 


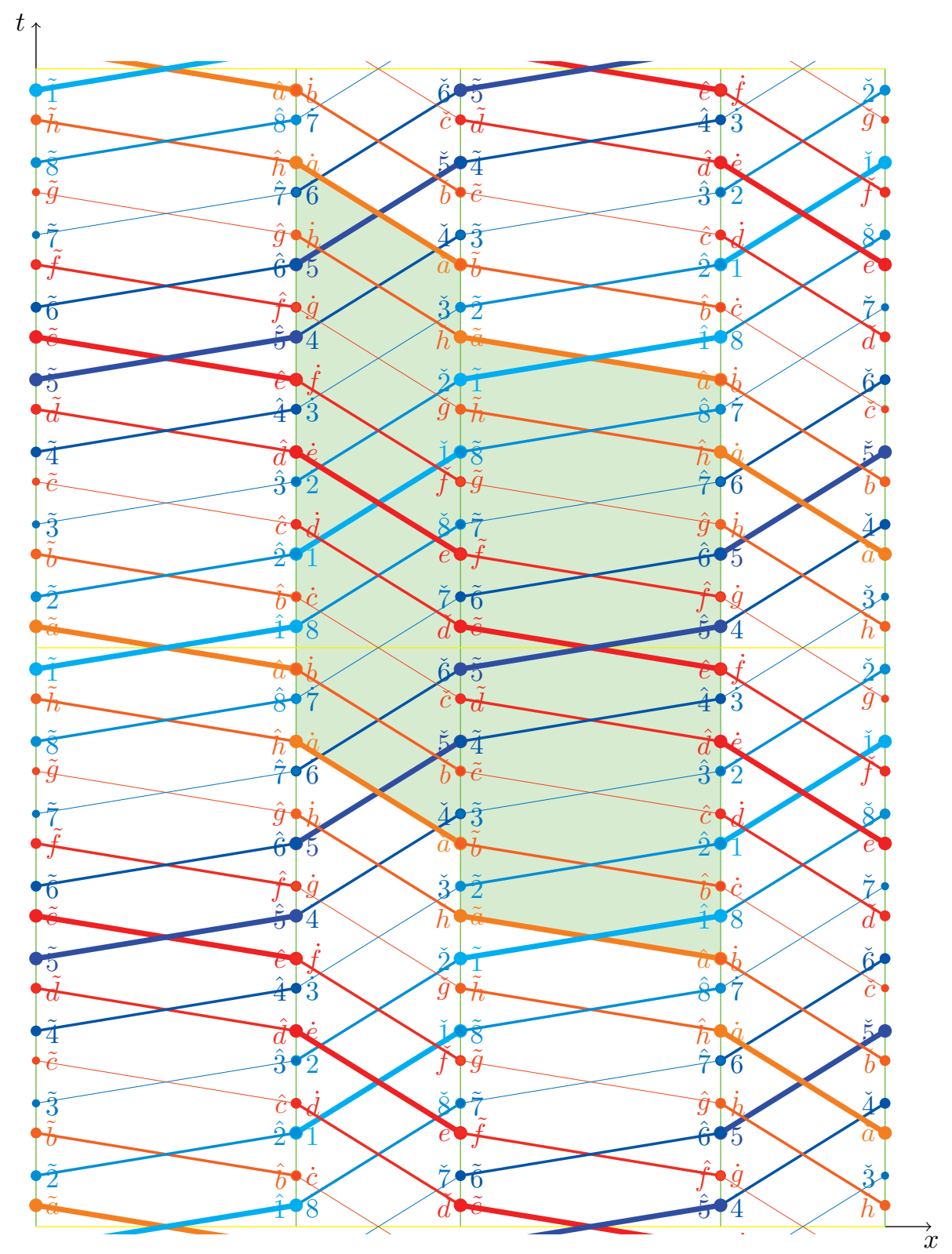

FIG. 2. The linearized characteristic structure of periodic solutions in the $(x, t)$-plane.

The labels $a-h$ and 1-8 label only one Riemann invariant rather than a complete $(z, u)$-state, but we can approximately reconstruct the $(z, u)$-states by considering the relative $r$ and $s$ values of the labeled states. Doing so, we observe that the locus of states at each jump corresponds to a path around an ellipse, with different orientations at each jump. We thus see that the periodic structure of Figure 2 is consistent with the ellipses drawn in Figure 3. The four ellipses drawn there represent the solution as a function of time at the corresponding entropy jump, and the circular arrows 

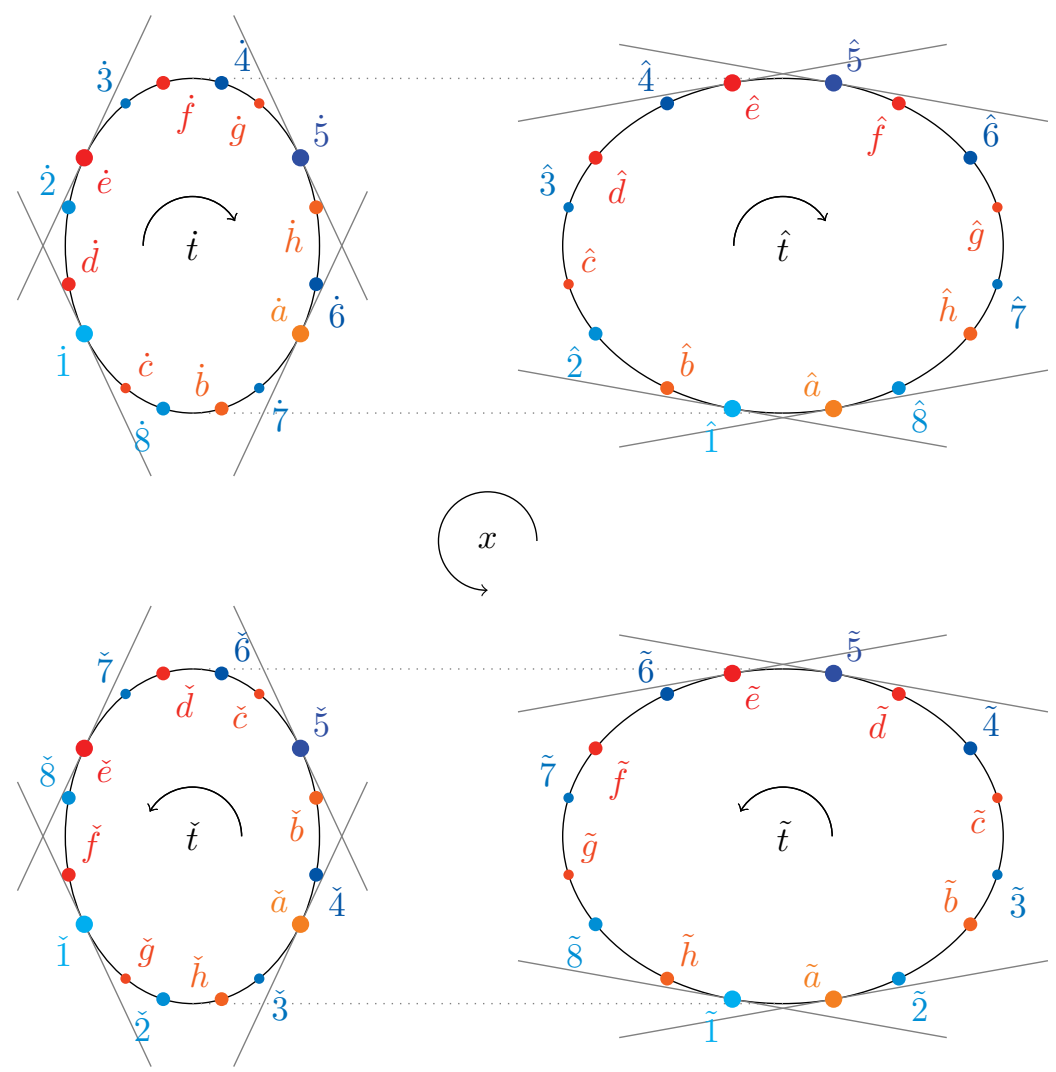

FIG. 3. Ellipses showing periodicity in the $(z, u)$-plane.

represent the direction of increase of $t$ and $x$ in the characteristic picture Figure 2 . Rotating anticlockwise around Figure 3 represents evolution (with respect to $x$ ) from $\dot{U}$ in the narrow strip to $\check{U}$, entropy jump to $\tilde{U}$, evolution in the wide strip to $\hat{U}$, and entropy jump back to a time-shift of $\dot{U}$.

Although we have presented these pictures of the solution as approximate, we show in this paper that the pictures become exact at the linearized level; that is, if we linearize the compressible Euler equations around a piecewise constant stationary solution, then the solutions given in Figures 2 and 3 are exact periodic solutions to the linearized Euler equations, with piecewise constant varying entropy, which balance compression and rarefaction.

3. The linear and nonlinear eigenvalue problems. In this section we reformulate the problem of existence of a periodic solution of the compressible Euler equations having the structure of Figure 2 as a nonlinear eigenvalue (fixed point) problem. In the next section we find exact solutions of the corresponding linearized eigenvalue problem. In subsequent sections we calculate the full spectrum of the linearized problem and discuss the issue of perturbation of the linearized solution as a way of obtaining periodic solutions of the nonlinear problem.

The periodic structure in Figure 2 is supported on an entropy field that oscillates between two different values. Moreover, it is apparent from Figure 2 that the solution, starting at $x=0$, returns after a nonlinear evolution in $x$ at the first entropy level, 
followed by a jump to the second entropy level, followed by a nonlinear evolution in $x$ at the second entropy level, followed by a jump back to the first entropy level, followed by a half period shift in $t$. We now formulate this precisely as a fixed point problem.

So consider a smooth solution $U(x, t)=(z(x, t), u(x, t))$ of the compressible Euler equations evolving through an entropy field that oscillates between two values $\bar{S}, \bar{m}$ and $\underline{S}, \underline{m}$, where $\bar{S}>\underline{S}, \bar{m}>\underline{m}$; cf. (10). Fix the widths $\underline{x}, \bar{x}>0$ of the entropy levels and assume $m=\bar{m}$ for $0<x<\bar{x}$, and $m=\underline{m}$ for $\bar{x}<x<\bar{x}+\underline{x}$, and then continue the entropy field periodically in $x$; cf. Figure 4 . Let $U(x, t)=\bar{U}(x, t) \equiv(\bar{z}(x, t), \bar{u}(x, t))$ in $0<x<\bar{x}$, so that $\bar{U}(x, t)$ solves

$$
\begin{array}{r}
z_{t}+\frac{c(z)}{\bar{m}} u_{x}=0, \\
u_{t}+\bar{m} c(z) z_{x}=0 ;
\end{array}
$$

and let $U(x, t)=\underline{U}(x, t) \equiv(\underline{z}(x, t), \underline{u}(x, t))$ in $\bar{x}<x<\bar{x}+\underline{x}$, so that $\underline{U}(x, t)$ solves

$$
\begin{aligned}
z_{t}+\frac{c(z)}{\underline{m}} u_{x} & =0, \\
u_{t}+\underline{m} c(z) z_{x} & =0 .
\end{aligned}
$$

Thus to demonstrate the existence of a solution of the compressible Euler equations with periodic sound wave propagation it would suffice to prove that there exists such a smooth solution defined on $0<x<\bar{x}+\underline{x}$ such that it continues globally to a periodic solution. Assume that $U(x, t)$ is a weak solution so that the Rankine-Hugoniot jump conditions (13) hold at $x=\bar{x}$ and $x=\bar{x}+\underline{x}$. This says that $(\bar{z}(\bar{x}-, t), \bar{u}(\bar{x}-, t), \bar{m})$ and $(\underline{z}(\bar{x}+, t), \underline{u}(\bar{x}+, t), \underline{m})$ lie on the same contact discontinuity wave curve of the 2 -family; cf. [23]. The jump conditions yield

$$
\begin{aligned}
\bar{u} & =\underline{u}, \\
\underline{z} & =\bar{z}\left(\frac{\bar{m}}{\underline{m}}\right)^{\frac{\gamma-1}{\gamma}}, \\
\underline{m} \underline{z} & =\bar{m} \bar{z}\left(\frac{\bar{m}}{\underline{m}}\right)^{\frac{-1}{\gamma}}, \\
\underline{c} & =\bar{c}\left(\frac{\bar{m}}{\underline{m}}\right)^{\frac{1}{\gamma}} .
\end{aligned}
$$

Now, consistent with Figure 2, set $\dot{U}(t)=U(0+, t), \check{U}(t)=U(\bar{x}-, t), \tilde{U}(t)=U(\bar{x}+, t)$, and $\hat{U}(t)=U(\bar{x}+\underline{x}-, t)$. Based on this, define the nonlinear evolution operators $\overline{\mathcal{E}}$, $\underline{\mathcal{E}}$, and the jump operators $\mathcal{J}, \mathcal{J}^{-1}$ by

$$
\begin{aligned}
\check{U} & =\overline{\mathcal{E}} \dot{U}, \\
\tilde{U} & =\mathcal{J} \check{U}, \\
\hat{U} & =\underline{\mathcal{E}} \tilde{U},
\end{aligned}
$$

and define the extended function $U^{*}$ by

$$
U^{*}=\mathcal{J}^{-1} \hat{U}
$$

Then $\overline{\mathcal{E}}$ and $\underline{\mathcal{E}}$ represent evolution in space by the nonlinear wave operators (17) and (18) across the respective entropy levels, and $\mathcal{J}, \mathcal{J}^{-1}$ are the jump operators 
determined by the Rankine-Hugoniot jump conditions (13), (14). That $\mathcal{J}^{-1}$ is the inverse of $\mathcal{J}$ follows from the invariance of the jump conditions under the interchange of $U_{L}$ and $U_{R}$. It follows from (19) that $\mathcal{J}$ is a linear operator when the equation of state is taken to be polytropic.

To formulate the eigenvalue problem that captures the periodic structure of Figure 2 , restrict the initial data $\dot{U}(t)$ to $2 \pi$-periodic functions at $x=0$, and impose the periodicity condition

$$
\dot{U}=\mathcal{S} U^{*}
$$

where $\mathcal{S}$ denotes the half period shift operator defined by

$$
[\mathcal{S} U](t)=U(t+\pi) .
$$

From this we conclude that the eigenvalue problem that imposes the periodicity structure of Figure 2 is $^{2}$

$$
\mathcal{S} \cdot \mathcal{J}^{-1} \cdot \underline{\mathcal{E}} \cdot \mathcal{J} \cdot \overline{\mathcal{E}} \dot{U}=\dot{U} .
$$

3.1. Nondimensionalization. We now recast (22) in a nondimensional form by introducing dimensionless variables $(w, v)$ in place of $(z, u)$. To this end, we first restrict ourselves to smooth solutions $U(x, t)=(z(x, t), u(x, t))$ of the compressible Euler equations defined at constant entropy $S \equiv S_{0}, m \equiv m_{0}$, in a region $x \geq x_{0}$. Let $z_{0}$ and $u_{0}$ be base states from which values of $z$ and $u$ are measured, respectively, and set $c_{0}=c\left(m_{0}, z_{0}\right)$ equal to the sound speed at $\left(z_{0}, m_{0}\right)$; cf. (12). Give time and space the same dimension by defining $y$ through the relation

$$
y-y_{0}=\frac{x-x_{0}}{c_{0}},
$$

so that equations (15) take on the dimensionless form

$$
\begin{aligned}
& \left(\frac{z}{z_{0}}\right)_{t}+\frac{c(z)}{c_{0}}\left(\frac{u}{m_{0} z_{0}}\right)_{y}=0, \\
& \left(\frac{u}{m_{0} z_{0}}\right)_{t}+\frac{c(z)}{c_{0}}\left(\frac{z}{z_{0}}\right)_{y}=0,
\end{aligned}
$$

in the region $y \geq y_{0}$. Based on (24), define the dimensionless variables

$$
\begin{aligned}
w & =\frac{z}{z_{0}}, \\
v & =\frac{u-u_{0}}{m_{0} z_{0}},
\end{aligned}
$$

and let

$$
\sigma=\frac{c\left(z_{0}\right)}{c(z)}=\frac{z_{0}^{d}}{z^{d}}=w^{-d} \equiv \sigma(w),
$$

where we have used $c(z, m)=K_{c} m z^{d}$ with

$$
d \equiv \frac{\gamma+1}{\gamma-1}
$$

\footnotetext{
${ }^{2}$ Note that the half period shift really just imposes a symmetry, as its effect over four entropy levels could be reproduced by a second application $\mathcal{J}^{-1} \cdot \underline{\mathcal{E}} \cdot \mathcal{J} \cdot \overline{\mathcal{E}}$.
} 
cf. (12). Thus the nonlinear equations (24) have the nondimensional form

$$
\begin{aligned}
& w_{y}+\sigma(w) v_{t}=0, \\
& v_{y}+\sigma(w) w_{t}=0,
\end{aligned}
$$

where $y$ is now the evolution variable.

Across an entropy jump between two constant values $\bar{m}$ and $\underline{m}$ with base states $\bar{z}_{0}$ and $\underline{z}_{0}$, respectively, the jump conditions are

$$
\begin{aligned}
& {[u]=\overline{m z}_{0} \bar{v}-\underline{m z}_{0} \underline{v}=0,} \\
& {[p]=\bar{m}^{2} \bar{z}^{d+1}-\underline{m}^{2} \underline{z}^{d+1}=0 .}
\end{aligned}
$$

So assuming the base states $\bar{z}_{0}$ and $\underline{z}_{0}$ satisfy the jump condition (30) as well, we find that the jump conditions in dimensionless variables become

$$
\begin{aligned}
\bar{w} & =\underline{w}, \\
\bar{m}^{\frac{d-1}{d+1}} \bar{v} & =\underline{m}^{\frac{d-1}{d+1}} \underline{v},
\end{aligned}
$$

the latter following from (30) using the jump relation for the base states in the form

$$
\frac{\bar{z}_{0}}{\underline{z}_{0}}=\left(\frac{\bar{m}}{\underline{m}}\right)^{\frac{-2}{d+1}} .
$$

In particular, (31) implies that $\sigma(w)$ is continuous across entropy jumps.

Now consider the nonlinear problem for smooth solutions evolving through two entropy levels $\bar{m}, \underline{m}$, of widths $\bar{x}, \underline{x}$, with base states $\bar{z}_{0}, \underline{z}_{0}$, extended periodically in $x$ starting with $\bar{m}$ in $0<x<\bar{x}$ and $\underline{m}$ in $\bar{x}<x<\bar{x}+\underline{x}$. Define $y=y(x)$ as the unique piecewise linear Lipschitz continuous function of $x$ such that $y(0)=0$, and such that (23) holds at each entropy level. That is, such that $y(0)=0, d y / d x=1 / \bar{c}$ in each $\bar{m}$ level, and $d y / d x=1 / \underline{c}$ in each $\underline{m}$ level. (We need only that $U$ is a smooth solution on the first two entropy levels in order to pose the periodicity condition.) The function $y(x)$ is graphed in Figure 5. Assuming this, the upperbar entropy level $0<x<\bar{x}$ goes over to $0<y<\bar{\theta}$,

$$
\bar{\theta}=\frac{\bar{x}}{c\left(\bar{z}_{0}\right)},
$$

the lowerbar entropy level goes over to $\bar{\theta}<y<\bar{\theta}+\underline{\theta}$,

$$
\underline{\theta}=\frac{\underline{x}}{c\left(\underline{z}_{0}\right)}
$$

and the nonlinear operators $\overline{\mathcal{E}}$ and $\underline{\mathcal{E}}$, expressed in dimensionless variables $w, v$, reduce simply to evolution in $y$ by system (29); cf. Figures 4, 5, and 6 .

Thus let $V=(w, v)$ denote the dimensionless variables, and define the evolution operator $\mathcal{E}(\theta)$ as evolution by system (29) through a $y$-interval of length $\theta$. That is,

$$
\mathcal{E}(\theta) V(0, \cdot)=V(\theta, \cdot)
$$

where $V(y, t)$ is the unique solution of the Cauchy problem for system (29) with Cauchy data $V(0, \cdot)$. Define also the entropy jump operator $\mathcal{J}$ acting on $V$ pointwise by

$$
\mathcal{J}\left[\begin{array}{l}
w \\
v
\end{array}\right]=\left(\begin{array}{ll}
1 & 0 \\
0 & J
\end{array}\right)\left[\begin{array}{l}
w \\
v
\end{array}\right]
$$




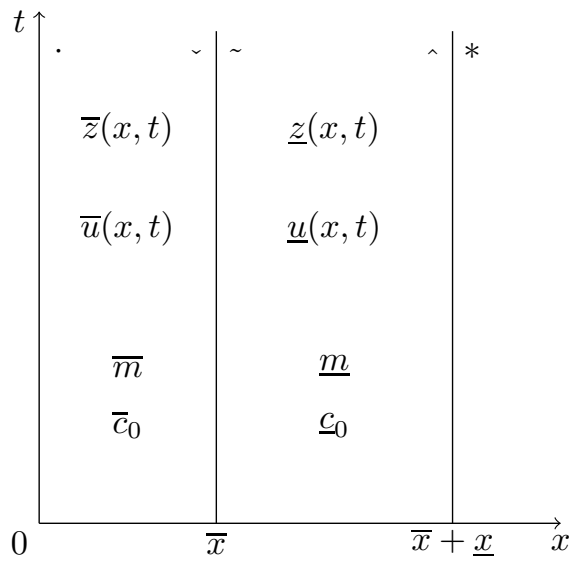

FIG. 4. Solutions at two constant entropy levels $\bar{S}, \underline{S}$ in the $(x, t)$-plane.

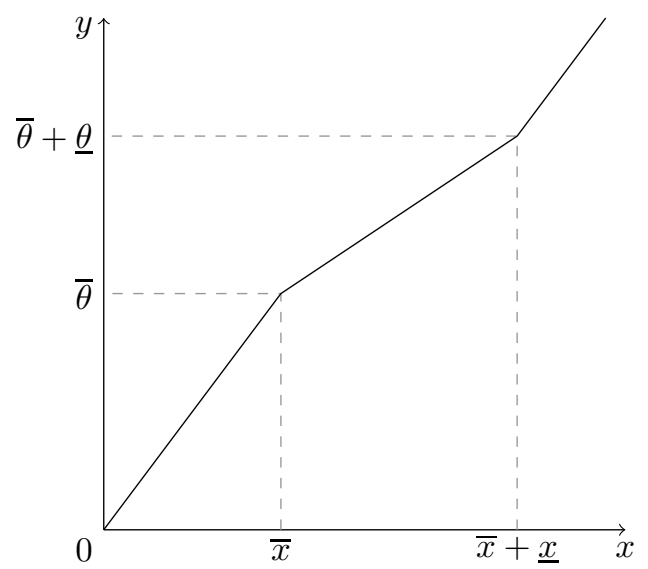

Fig. 5. The mapping $x \rightarrow y, \bar{x} \rightarrow \bar{\theta}, \underline{x} \rightarrow \underline{\theta}$.

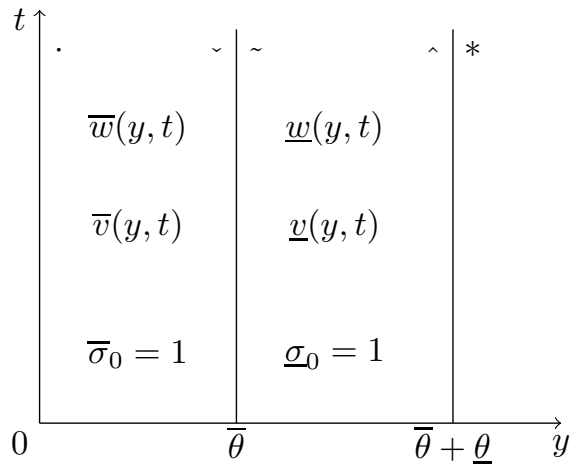

FIG. 6. Solutions at two constant entropy levels $\bar{S}, \underline{S}$ in the $(y, t)$-plane. 
where

$$
J=\left(\frac{\bar{m}}{\underline{m}}\right)^{\frac{d-1}{d+1}} ;
$$

cf. (31). Finally, define the shift operator $\mathcal{S}$ acting on $V$ by

$$
\mathcal{S} V(t)=V(t+\pi)
$$

Note that $\mathcal{E}(\theta)$ is nonlinear, but $\mathcal{J}$ and $\mathcal{S}$ are both linear operators when $\bar{m}$ and $\underline{m}$ are assumed to be fixed.

THEOREM 2. For fixed positive real numbers $\bar{\theta}, \underline{\theta}$, and $J$, define the nonlinear operator $\mathcal{N} \equiv \mathcal{N}(\bar{\theta}, \underline{\theta}, J)$ by

$$
\mathcal{N} \equiv \mathcal{S} \cdot \mathcal{J}^{-1} \cdot \mathcal{E}(\underline{\theta}) \cdot \mathcal{J} \cdot \mathcal{E}(\bar{\theta})
$$

and let $V(t)=(w(t), v(t))$ denote any smooth solution of

$$
\mathcal{N} V(\cdot)=V(\cdot)
$$

that satisfies the average one and zero average conditions

$$
w_{0} \equiv \frac{1}{2 \pi} \int_{0}^{2 \pi} w(t) d t=1
$$

and

$$
v_{0} \equiv \frac{1}{2 \pi} \int_{0}^{2 \pi} v(t) d t=0
$$

respectively. Then given any base state $U_{0}=\left(\bar{z}_{0}, \bar{u}_{0}\right)$ and entropy state $\bar{m}$, there is a periodic solution $U(x, t)=(z(x, t), u(x, t))$ of $(11)$, determined uniquely by $V(t)$, with average values

$$
\frac{1}{2 \pi} \int_{0}^{2 \pi} z(0, t) d t=\bar{z}_{0}
$$

and

$$
\frac{1}{2 \pi} \int_{0}^{2 \pi} u(0, t) d t=\bar{u}_{0} .
$$

Proof. Given the states $\bar{z}_{0}, \bar{u}_{0}$, and $\bar{m}$, and the parameters $\bar{\theta}, \underline{\theta}$, and $J$, define

$$
\begin{aligned}
& \underline{u}_{0}=\bar{u}_{0}, \\
& \underline{m}=\bar{m} J^{-\frac{d+1}{d-1}},
\end{aligned}
$$

and

$$
\underline{z}_{0}=\left(\frac{\bar{m}}{\underline{m}}\right)^{\frac{2}{d+1}} \bar{z}_{0} .
$$

Set the values of $\bar{x}$ and $\underline{x}$ equal to

$$
\begin{aligned}
& \bar{x}_{0}=c\left(\bar{z}_{0}\right) \bar{\theta}, \\
& \underline{x}_{0}=c\left(\underline{z}_{0}\right) \underline{\theta} .
\end{aligned}
$$


Now define the nonlinear evolution operator

$$
V(y, \cdot)=\mathcal{N}(y)[V(\cdot)] \equiv \begin{cases}\mathcal{E}(y)[V(\cdot)], & 0 \leq y<\bar{\theta} \\ \mathcal{E}(y-\bar{\theta}) \mathcal{J} \mathcal{E}(\bar{\theta})[V(\cdot)], & 0<y-\bar{\theta}<\underline{\theta}, \\ \mathcal{S} \mathcal{J}^{-1} \mathcal{E}(\underline{\theta}) \mathcal{J} \mathcal{E}(\bar{\theta})[V(\cdot)], & y=\bar{\theta}+\underline{\theta} .\end{cases}
$$

By this definition, $\mathcal{N}(y)[V(\cdot)]$ defines the evolution of initial data $V(t)$ through interval $[0, y)$ for the dimensionless nonlinear problem consisting of entropy jumps at $y=\bar{\theta}$ and $y=\bar{\theta}+\underline{\theta}$. Thus it follows directly from $(38)$ that $V(y, t)$ extends to a global periodic solution of the nondimensionalized equations having periodic tile $0<t<2 \pi$, $0<y<\bar{\theta}+\underline{\theta}$, with periodic motion vector $\mathbf{X}=(\bar{\theta}+\underline{\theta}, \pi)$. Thus defining $U(x, t)=$ $(z(x, t), u(x, t))$ by

$$
\begin{aligned}
& z=w z_{0}, \\
& u=u_{0}+m z_{0} v
\end{aligned}
$$

from our constructions above, $U(x, t)$ is a global periodic solution of (11) having periodic tile $0<t<2 \pi, 0<x<\bar{x}+\underline{x}$, with periodic motion vector $\mathbf{X}=(\bar{x}+\underline{x}, \pi)$. The averages (40), (41) follow directly from (43).

Note that the periodic motion vector $\mathbf{X}$ determines the "group velocities" of the solution: these are the effective propagation speeds of the max / min characteristics (which are discontinuous at the entropy jumps), given by

$$
c_{g}= \pm \frac{\bar{x}+\underline{x}}{\pi}
$$

The fact that the forward and backward group velocities are the same follows from the symmetry obtained by imposing a shift of exactly half a period. Because corresponding characteristics always jump forward in time, $c_{g}$ will be less than the mean characteristic speed. Thus we have dispersive type behavior, with different group and phase velocities, in a purely hyperbolic nonlinear system. To the best of our knowledge, this is the first discovery of such an effect in a fully nonlinear hyperbolic problem.

In summary, our fundamental nonlinear problem is the dimensionless eigenvalue problem (39), where the operator $\mathcal{N}$ is defined in (38) through the defining relations (34)-(37). Solutions of (39) correspond to periodic solutions of (11) in a neighborhood of any state $U_{0}, \bar{m}$ via the transformations (42)-(43).

4. Periodic solutions of the linearized problem. In this section we introduce the linearized eigenvalue problem associated with (39), and then characterize the solutions of the corresponding linearized operator depending on the parameters $\bar{\theta}$, $\underline{\theta}$ and $J$. In the next section we analyze the spectrum of the linearized operator, and introduce a nonresonance condition on the parameters that guarantees that exact solutions of the linearized eigenvalue problem are isolated in the kernel of the linearized operator. These exact solutions are depicted in Figures 2 and 3.

For the linearized eigenvalue problem, replace the nonlinear evolution operator $\mathcal{E}(\theta)$ by the linear operator $\mathcal{L}(\theta)$ obtained by setting $\sigma(w) \equiv 1$ in equations $(29) .{ }^{3}$ (By $(25),(27)$, in the dimensional problem this represents taking the sound speed equal to

\footnotetext{
${ }^{3}$ For example, $\mathcal{L}(\theta)$ is the operator obtained by linearizing $\mathcal{E}(\theta)$ about the constant state base state.
} 
the value of the sound speed at the $z$-base state at each entropy level; cf. (42)-(43).) Thus the linear evolution is

$$
\mathcal{L}(y) V(0, \cdot) \equiv V(y, \cdot)
$$

where $V(y, t)$ is the unique solution of the linear system

$$
\begin{aligned}
& w_{y}+v_{t}=0, \\
& v_{y}+w_{t}=0,
\end{aligned}
$$

with Cauchy data $V(0, t)$; cf. (29) and (34). Then the linearized eigenvalue problem associated with (39) is the problem

$$
\mathcal{M} V(\cdot)=V(\cdot)
$$

where $\mathcal{M} \equiv \mathcal{M}(\bar{\theta}, \underline{\theta}, J)$ is the linear operator defined by

$$
\mathcal{M} \equiv \mathcal{S} \cdot \mathcal{J}^{-1} \cdot \mathcal{L}(\underline{\theta}) \cdot \mathcal{J} \cdot \mathcal{L}(\bar{\theta})
$$

where again $\mathcal{J}$ and $\mathcal{S}$ are the linear jump and shift operators given by (36) and (37), respectively. We now find elements of the kernel of the linear operator $\mathcal{M}-\mathcal{I}$, and in the next section, we give conditions on the parameters that isolate these solutions in the kernel.

Our method is to extend the operator $\mathcal{M}$ to the complex plane, show that the operator splits orthogonally onto the two complex dimensional subspaces associated with each Fourier mode, and then analyze the kernel on each of these subspaces. The complex vector space associated with each Fourier mode has no real representatives, so the eigenvalue problem is two complex dimensional, equivalent to a four-dimensional real problem. To solve this, we introduce a representation of each Fourier mode in terms of which the complex eigenvalue problem reduces to a two-dimensional real problem, which we can solve in closed form. We recover the real solutions by adding the complex solution in the 1-mode to its complex conjugate, which lives in the -1 mode.

So fix values of $\bar{\theta}, \underline{\theta}$, and $J$, and consider the corresponding operator $\mathcal{M}$ determined in (48). Let $V_{0}(t)=(w(t), v(t))$ be a $2 \pi$-periodic function in the domain of $\mathcal{M}$. For example, $V_{0}(t)$ serves as initial data at $y=0$ for the linear evolution in $y$ associated with $\mathcal{L}(\theta)$. Using this, let $V(y, \cdot)$ be the function obtained at $y \in(0, \bar{\theta}+\underline{\theta})$ in the construction of $\mathcal{M} V_{0}(t)$ according to (48) - that is, $V(y, \cdot)=\mathcal{L}(y) V_{0}$ for $0<y<\bar{\theta}$; $V(y, \cdot)=\mathcal{L}(y-\bar{\theta}) \mathcal{J} \mathcal{L}(\bar{\theta}) V_{0}$ for $\bar{\theta}<y<\bar{\theta}+\underline{\theta}$ - and define the following boundary functions:

$$
\begin{aligned}
\dot{V}(t) & =V(0+, t), \\
\breve{V}(t) & =V(\bar{\theta}-, t), \\
\tilde{V}(t) & =V(\bar{\theta}+, t) \\
\hat{V}(t) & =V(\bar{\theta}+\underline{\theta}-, t),
\end{aligned}
$$

and

$$
V^{*}(t)=\mathcal{J}^{-1} \hat{V}(t)=V(\bar{\theta}+\underline{\theta}+, t) ;
$$

cf. (20)-(21) and Figure 2. Thus the linear eigenvalue equation (47) can be reexpressed as

$$
\mathcal{S} V^{*}(t)=\dot{V}(t)
$$


We will let $V$ refer to $V(t)$ or $V(y, t)$ according to the context.

Note now that $\mathcal{M}$ extends naturally to complex valued $2 \pi$-periodic functions $V(t)$. Indeed, the linear evolution $\mathcal{L}(\theta)$ and jump operator $\mathcal{J}$ can both be applied to complex valued functions for every positive real $\theta$. Thus assume that $V(t)$ is complex valued. The complex $2 \pi$-periodic functions $V(t)$ have the Fourier expansion

$$
V(t)=\left[\begin{array}{c}
w(t) \\
v(t)
\end{array}\right]=\sum_{n=-\infty}^{+\infty} V_{n} e^{i n t} \in \Sigma,
$$

where we take

$$
V_{n}=\left[\begin{array}{c}
a_{n} \\
-i b_{n}
\end{array}\right]
$$

with $a_{n}$ and $b_{n}$ arbitrary complex numbers, $a_{n}, b_{n} \in \mathbb{C}$, and

$$
\Sigma \equiv L^{2}[0,2 \pi) \times L^{2}[0,2 \pi) .
$$

We place the $-i$ factor on $b_{n}$ in (51) for convenience. The expression (50) gives an expression for $w(t)$ and $v(t)$ in terms of the orthonormal basis

$$
\left\{\left[\begin{array}{l}
1 \\
0
\end{array}\right] e^{i n t},\left[\begin{array}{l}
0 \\
1
\end{array}\right] e^{i n t}\right\}_{n=-\infty}^{+\infty}
$$

for the $2 \pi$-periodic, square integrable complex valued functions $\left[\begin{array}{l}w(t) \\ v(t)\end{array}\right]$ defined on $0 \leq t<2 \pi$. That is, let

$$
\Sigma_{n} \equiv \operatorname{Span}\left\{\left[\begin{array}{l}
1 \\
0
\end{array}\right] e^{i n t},\left[\begin{array}{l}
0 \\
1
\end{array}\right] e^{i n t}\right\}
$$

so that

$$
\Sigma=\bigoplus_{n=-\infty}^{+\infty} \Sigma_{n}
$$

gives an orthogonal decomposition of $\Sigma$ with respect to the $L^{2}$-inner product

$$
\left\langle\left[\begin{array}{c}
w_{1}(\cdot) \\
v_{1}(\cdot)
\end{array}\right],\left[\begin{array}{c}
w_{2}(\cdot) \\
v_{2}(\cdot)
\end{array}\right]\right\rangle=\frac{1}{2 \pi} \int_{0}^{2 \pi}\left(w_{1}(t), v_{1}(t)\right) \cdot \overline{\left(w_{2}(t), v_{2}(t)\right)} d t .
$$

Note that each $\Sigma_{n}$ is a complex two-dimensional vector space, and that

$$
V(t)=\sum_{n=-\infty}^{\infty} V_{n} e^{i n t}
$$

represents a real function if and only if

$$
\overline{V_{-n}}=V_{n} .
$$

We now show that $\mathcal{M}$ factors over the decomposition (52).

We first obtain a matrix representation for the linear evolution operator $\mathcal{L}(\theta)$ of system (46) that applies to complex valued functions $V(t)$. 
LEMMA 3. Let

$$
V(y, t)=\left[\begin{array}{l}
w(y, t) \\
v(y, t)
\end{array}\right]
$$

denote the complex valued solution of (46) starting from data $V_{0}(t)=V(0, t)=$ $V_{n} e^{i n t} \in \Sigma_{n}$, where $V_{n}$ is given in (51). Then

$$
\begin{aligned}
V(y, t) & =\frac{1}{2}\left(\begin{array}{cc}
1 & 1 \\
1 & -1
\end{array}\right)\left(\begin{array}{cc}
e^{-i n y} & 0 \\
0 & e^{i n y}
\end{array}\right)\left(\begin{array}{cc}
1 & 1 \\
1 & -1
\end{array}\right) V(y, 0) \\
& =\left(\begin{array}{cc}
\cos (n y) & -i \sin (n y) \\
-i \sin (n y) & \cos (n y)
\end{array}\right) V_{n} e^{i n t} \in \Sigma_{n} .
\end{aligned}
$$

Proof. Adding and subtracting (46) gives the equivalent system

$$
\begin{aligned}
& (v+w)_{y}+(v+w)_{t}=0 \\
& (v-w)_{y}-(v-w)_{t}=0 .
\end{aligned}
$$

Since these are scalar wave equations, it follows that

$$
(v+w)(y, t)=\left(v_{0}+w_{0}\right)(t-y)=e^{-i n y}\left(v_{0}+w_{0}\right)(t)
$$

and

$$
(v-w)(y, t)=(v-w)(t+y)=e^{i n y}\left(v_{0}-w_{0}\right)(t) .
$$

Writing this in matrix form yields (55).

From Lemma 3 it follows that $\Sigma_{n}$ is an invariant subspace for $\mathcal{L}(\theta)$, and so $\mathcal{L}(\theta)$ respects the orthogonal decomposition (52). Moreover, since evolution by system (46) takes real functions to real functions, it follows that $\mathcal{L}(\theta)$ does as well. Moreover, if $V(t)=P(t)+i Q(t)$, where $P$ and $Q$ are the real and imaginary parts of $V$, then

$$
\mathcal{L}(\theta) V(\cdot)=\mathcal{L}(\theta) P(\cdot)+i \mathcal{L} Q(\cdot)
$$

where on $P$ and $Q, \mathcal{L}(\theta)$ reduces to real evolution. We next show that $\mathcal{M}$ also respects the decomposition (52), and get an expression for $\mathcal{M}$ in each subspace $\Sigma_{n}$.

To this end, let $\mathcal{T}_{n}: \mathbb{C}^{2} \rightarrow \Sigma_{n}$ be the representation of $\Sigma_{n}$ defined by

$$
\mathcal{T}_{n}\left[\begin{array}{l}
a \\
b
\end{array}\right]=\left[\begin{array}{c}
a \\
-i b
\end{array}\right] e^{i n t}
$$

and write

$$
\left[\begin{array}{l}
w_{0}(t) \\
v_{0}(t)
\end{array}\right]=\sum_{-\infty}^{+\infty}\left[\begin{array}{c}
a_{n} \\
-i b_{n}
\end{array}\right] e^{i n t}=\sum_{-\infty}^{+\infty} \mathcal{T}_{n}\left[\begin{array}{c}
a_{n} \\
b_{n}
\end{array}\right]
$$

LEMMA 4. The following formulas hold:

$$
\begin{aligned}
\mathcal{L}(\theta) \mathcal{T}_{n}\left[\begin{array}{l}
a_{n} \\
b_{n}
\end{array}\right]=\mathcal{T}_{n} R(n \theta)\left[\begin{array}{l}
a_{n} \\
b_{n}
\end{array}\right], \\
\mathcal{J} \mathcal{T}_{n}\left[\begin{array}{l}
a_{n} \\
b_{n}
\end{array}\right]=\mathcal{T}_{n} D\left[\begin{array}{c}
a_{n} \\
b_{n}
\end{array}\right] \\
\mathcal{S} \mathcal{T}_{n}\left[\begin{array}{l}
a_{n} \\
b_{n}
\end{array}\right]=(-1)^{n} \mathcal{T}_{n}\left[\begin{array}{l}
a_{n} \\
b_{n}
\end{array}\right],
\end{aligned}
$$


where $R(\theta)$ denotes real counterclockwise rotation through angle $\theta$,

$$
R(\theta)=\left(\begin{array}{cc}
\cos (\theta) & -\sin (\theta) \\
\sin (\theta) & \cos (\theta)
\end{array}\right)
$$

and $D \equiv D(J)$ denotes the diagonal $2 \times 2$ real matrix

$$
D=\left(\begin{array}{ll}
1 & 0 \\
0 & J
\end{array}\right)
$$

with

$$
J=\left(\frac{\bar{m}}{\underline{m}}\right)^{\frac{d-1}{d+1}}=\left(\frac{\bar{m}}{\underline{m}}\right)^{\frac{1}{\gamma}}
$$

cf. (35), (28), (27).

Proof. Equation (57) follows directly from (55), and (58), (59) follow directly from (35), (37), where in (59) we use

$$
\mathcal{S}\left[\begin{array}{c}
a_{n} \\
-i b_{n}
\end{array}\right] e^{i n t}=\left[\begin{array}{c}
a_{n} \\
-i b_{n}
\end{array}\right] e^{i n(t-\pi)} .
$$

Corollary 5. All of the operators in the decomposition (48) of $\mathcal{M}$ respect the decomposition (52).

Proof. To show that a linear operator respects (52), it suffices to show that $\Sigma_{n}$ is invariant for each $n$. This follows directly from (57)-(59).

It follows that $\mathcal{M}$ respects (52). This is made explicit in the next theorem, which also implies that the complex eigenvalue problem (47) reduces to a $2 \times 2$ real eigenvalue problem in each subspace $\Sigma_{n}$.

THEOREM 6.

$$
\mathcal{M}\left(\sum_{-\infty}^{+\infty} \mathcal{T}_{n}\left[\begin{array}{l}
a_{n} \\
b_{n}
\end{array}\right]\right)=\sum_{-\infty}^{+\infty} \mathcal{T}_{n} M_{n}\left[\begin{array}{l}
a_{n} \\
b_{n}
\end{array}\right]
$$

where $M_{n}=M_{n}(\bar{\theta}, \underline{\theta}, J)$ is the real $2 \times 2$ matrix given by

$$
M_{n}=(-1)^{n} M(n \bar{\theta}, n \underline{\theta}, J),
$$

with

$$
M(\bar{\theta}, \underline{\theta}, J) \equiv M=D(J)^{-1} R(\underline{\theta}) D(J) R(\bar{\theta}),
$$

where $R(\theta)$ and $D(J)$ are the real matrices given in (60), (61), and (62).

Proof. It follows from (57)-(59) that each $\Sigma_{n}$ is an invariant subspace for $\mathcal{L}(y), \mathcal{J}$, and $\mathcal{S}$, and hence is also for $\mathcal{M}$, which is a composition of these operators. Moreover, 
applying (57)-(59) we have

$$
\begin{aligned}
\mathcal{M}\left(\mathcal{T}_{n}\left[\begin{array}{l}
a \\
b
\end{array}\right]\right) & =\mathcal{S} \cdot \mathcal{J}^{-1} \cdot \mathcal{L}(\underline{\theta}) \cdot \mathcal{J} \cdot \mathcal{L}(\bar{\theta}) \cdot \mathcal{T}_{n}\left[\begin{array}{l}
a \\
b
\end{array}\right] \\
& =\mathcal{S} \cdot \mathcal{J}^{-1} \cdot \mathcal{L}(\underline{\theta}) \cdot \mathcal{J} \cdot \mathcal{T}_{n} R(n \bar{\theta})\left[\begin{array}{l}
a \\
b
\end{array}\right] \\
& =\mathcal{S} \cdot \mathcal{J}^{-1} \cdot \mathcal{L}(\underline{\theta}) \cdot \mathcal{T}_{n} D R(n \bar{\theta})\left[\begin{array}{l}
a \\
b
\end{array}\right] \\
& =\mathcal{S} \cdot \mathcal{T}_{n} D^{-1} R(n \underline{\theta}) D R(n \bar{\theta})\left[\begin{array}{l}
a \\
b
\end{array}\right] \\
& =\mathcal{T}_{n}(-1)^{n} D^{-1} R(n \underline{\theta}) D R(n \bar{\theta})\left[\begin{array}{l}
a \\
b
\end{array}\right] \\
& =\mathcal{T}_{n} M_{n}\left[\begin{array}{l}
a \\
b
\end{array}\right],
\end{aligned}
$$

where $M_{n}$ is the real $2 \times 2$ matrix

$$
M_{n}=(-1)^{n} D^{-1} R(n \underline{\theta}) D R(n \bar{\theta})=(-1)^{n} M(n \bar{\theta}, n \underline{\theta}, J) .
$$

This verifies (63), (64), and (65), and completes the proof of the theorem. Note that $D(J)$ and $R(\theta)$ do not, in general, commute.

In summary, Theorem 6 implies that $\mathcal{M}(\theta)$ respects the orthogonal decomposition (52), takes real functions to real functions, and

$$
\mathcal{M}(\theta) V(\cdot)=\mathcal{M}(\theta) P(\cdot)+i \mathcal{M} Q(\cdot)
$$

if $V=P+i Q$ for $P$ and $Q$ real.

Consider now the linear eigenvalue problem (47) for a $2 \pi$-periodic function $V(t)$. Let

$$
V=\sum_{n \in \mathbb{Z}} V_{n} e^{i n t}=\sum_{n \in \mathbb{Z}} \mathcal{T}_{n}\left[\begin{array}{l}
a_{n} \\
b_{n}
\end{array}\right]
$$

denote the expansion of $V$ into Fourier modes, where $V_{n}$ is given in $(51), \mathcal{T}_{n}$ in (56).

THEOREM 7. The function $V(t)$ solves the linear eigenvalue problem (47) if and only if

$$
(-1)^{n} M(n \bar{\theta}, n \underline{\theta}, J)\left[\begin{array}{l}
a_{n} \\
b_{n}
\end{array}\right]=\left[\begin{array}{l}
a_{n} \\
b_{n}
\end{array}\right]
$$

for every $n \in \mathbb{Z}$.

Proof. Let $V$ have the decomposition (66), and assume $\mathcal{M} V=V$. Then

$$
\begin{aligned}
\sum_{n \in \mathbb{Z}} \mathcal{T}_{n}\left[\begin{array}{l}
a_{n} \\
b_{n}
\end{array}\right] & =V=\mathcal{M} V \\
& =\mathcal{M}\left(\sum_{n \in \mathbb{Z}} \mathcal{T}_{n}\left[\begin{array}{l}
a_{n} \\
b_{n}
\end{array}\right]\right) \\
& =\sum_{n \in \mathbb{Z}} \mathcal{T}_{n} M_{n}\left[\begin{array}{l}
a_{n} \\
b_{n}
\end{array}\right],
\end{aligned}
$$


where we have applied Theorem 6 . Thus, since the functions $e^{\text {int }}$ are independent, it follows that

$$
\mathcal{T}_{n}\left[\begin{array}{l}
a_{n} \\
b_{n}
\end{array}\right]=\mathcal{T}_{n} M_{n}\left[\begin{array}{l}
a_{n} \\
b_{n}
\end{array}\right]
$$

for every $n \in \mathbb{Z}$, so also

for all $n \in \mathbb{Z}$. Since

$$
M_{n}\left[\begin{array}{l}
a_{n} \\
b_{n}
\end{array}\right]=\left[\begin{array}{l}
a_{n} \\
b_{n}
\end{array}\right]
$$

$$
M_{n}=(-1)^{n} M(n \bar{\theta}, n \underline{\theta}, J),
$$

we have (67).

4.1. Matrix eigenvalue problems. Theorem 7 reduces the problem of finding solutions of the complex linear eigenvalue problems (47) to the problem of finding solutions $(a, b) \in \mathbb{R}^{2}$ of the $2 \times 2$ linear eigenvalue problems

$$
M_{n}\left[\begin{array}{l}
a \\
b
\end{array}\right]=\left[\begin{array}{l}
a \\
b
\end{array}\right],
$$

where $M_{n}$ is the real matrix $M_{n}=(-1)^{n} M(n \bar{\theta}, n \underline{\theta}, J)$. It follows that complex eigenvectors of $M_{n}$ can be rescaled to real eigenvectors, and so to solve (47) it suffices to characterize real solutions $(a, b)$ of (68). Thus for (68), it suffices to characterize the eigenspaces of $\pm M$ in terms of $(\bar{\theta}, \underline{\theta}, J)$. To this end, let $q=(a, b)^{t r}$, let $\|q\|=\sqrt{a^{2}+b^{2}}$ denote Euclidean norm, and let $\dot{q}, \check{q}, \tilde{q}, \hat{q}$, and $q^{*}$ denote the vector states determined by the decomposition (65) of $M$, so that

$$
\begin{aligned}
\dot{q} & =q, \\
\check{q} & =R(\bar{\theta}) \dot{q}, \\
\tilde{q} & =D \check{q}, \\
\hat{q} & =R(\underline{\theta}) \tilde{q}, \\
q^{*} & =D^{-1} \hat{q} ;
\end{aligned}
$$

cf. (49). Let $\dot{\mu}, \check{\mu}, \tilde{\mu}, \hat{\mu}$, and $\mu^{*}$ denote the angles these vectors make with the positive $x$-axis, respectively. Our problem then is to characterize solutions $q$ of the commutation condition

$$
M(n \bar{\theta}, n \underline{\theta}, J) q \equiv D^{-1}(J) R(n \underline{\theta}) D(J) R(n \bar{\theta}) q=(-1)^{n} q,
$$

in terms of the parameters $(\bar{\theta}, \underline{\theta}, J)$. Since $R(\theta)$ is the rotation through angle $\theta$ and $D(J)$ just scales the second coordinate $b$ by factor $J,(69)$ places very tight constraints on the possible angles $\dot{\mu}, \check{\mu}, \tilde{\mu}, \hat{\mu}$, and $\mu^{*}$, consistent with (69). The eigenvalues of $M$ are given in the following theorem.

TheOrem 8. Assume that $(\bar{\theta}, \underline{\theta})$ are real and that $J>1 ; c f$. (62). Then the eigenvalues of $M(\bar{\theta}, \underline{\theta}, J)$ are given by

$$
\lambda=\beta \pm \sqrt{\beta^{2}-1},
$$

where

$$
\beta=\cos (\underline{\theta}) \cos (\bar{\theta})-\frac{J^{2}+1}{2 J} \sin (\underline{\theta}) \sin (\bar{\theta}) .
$$


Proof. Observe that $\operatorname{det} M=1$, so the eigenvalues $\lambda$ of $M$ satisfy

$$
\lambda^{2}-2 \beta \lambda+1=0
$$

where $\beta=\frac{1}{2} \operatorname{tr} M$. Solutions are given by (70), and writing

$$
\begin{aligned}
M & =D^{-1} R(\underline{\theta}) D R(\bar{\theta}) \\
& =\left(\begin{array}{cc}
\cos (\underline{\theta}) & -\sin (\underline{\theta}) \\
\sin (\underline{\theta}) / J & \cos (\underline{\theta}) / J
\end{array}\right)\left(\begin{array}{cc}
\cos (\bar{\theta}) & -\sin (\bar{\theta}) \\
J \sin (\bar{\theta}) & J \cos (\bar{\theta})
\end{array}\right)
\end{aligned}
$$

and calculating the trace yields (71).

We now construct solutions of (69) that correspond to solutions in the eigenspaces $\Sigma_{-1}$ and $\Sigma_{1}$ that represent solutions of (47) with the periodic structure of Figures 2 and 3. For $n= \pm 1$, the problem (69) becomes, respectively,

$$
\begin{aligned}
M(\bar{\theta}, \underline{\theta}, J) q & =D^{-1}(J) R(\underline{\theta}) D(J) R(\bar{\theta}) q=-q, \\
M(-\bar{\theta},-\underline{\theta}, J) q & =D^{-1}(J) R(-\underline{\theta}) D(J) R(-\bar{\theta}) q=-q .
\end{aligned}
$$

We obtain the real solution below by adding the solution in $\Sigma_{-1}$ to the solution in $\Sigma_{1}$; cf. (54). Our purpose in the next section is to find nonresonance conditions on $(\bar{\theta}, \underline{\theta}, J)$ that isolate these in the kernel of $\mathcal{M}$.

To accurately model the widths of entropy levels in Figure 2, we impose the condition $\bar{\theta}+\underline{\theta}<\pi$. In particular, according to (44), this imposes a group velocity $(\bar{\theta}+\underline{\theta}) / \pi$, which is slower than the local wavespeed $\sigma_{0}=1$. The following theorem identifies the unique solution of (72) in $\Sigma_{1}$ and $\Sigma_{-1}$.

TheOrem 9. Assume that $J>1, \bar{\theta}>0, \underline{\theta}>0$, and

$$
\bar{\theta}+\underline{\theta}<\pi .
$$

Then $q$ is a solution of (72) if and only if

$$
J=\cot (\bar{\theta} / 2) \cot (\underline{\theta} / 2)
$$

and $q \in \operatorname{Span}\{\mathbf{q}\}$, where

$$
\mathbf{q}=(\cos (\bar{\theta} / 2),-\sin (\bar{\theta} / 2)) .
$$

Furthermore, if $\dot{q}=\mathbf{q}$, then also

$$
\begin{aligned}
\check{q} & =(\cos (\bar{\theta} / 2), \sin (\bar{\theta} / 2)), \\
\tilde{q} & =(\cos (\bar{\theta} / 2), J \sin (\bar{\theta} / 2)) \\
& =\alpha(\cos (\pi / 2-\underline{\theta} / 2), \sin (\pi / 2-\underline{\theta} / 2)), \\
\hat{q} & =(-\cos (\bar{\theta} / 2), J \sin (\bar{\theta} / 2)) \\
& =\alpha(-\cos (\pi / 2-\underline{\theta} / 2), \sin (\pi / 2-\underline{\theta} / 2)), \\
q^{*} & =(-\cos (\bar{\theta} / 2), \sin (\bar{\theta} / 2))=-q,
\end{aligned}
$$

where we have set $\alpha=\|\tilde{q}\|$. These states are diagrammed in Figure 7 .

Note that $J=\cot (\bar{\theta} / 2) \cot (\underline{\theta} / 2)>1$ when $\bar{\theta}+\underline{\theta}<\pi$ because the function $\cot (\theta)$ is decreasing on $0<\theta<\pi / 2$, so

$$
1=\cot (\bar{\theta} / 2) \tan (\bar{\theta} / 2)=\cot (\bar{\theta} / 2) \cot (\pi / 2-\bar{\theta} / 2)<\cot (\bar{\theta} / 2) \cot (\underline{\theta} / 2) .
$$




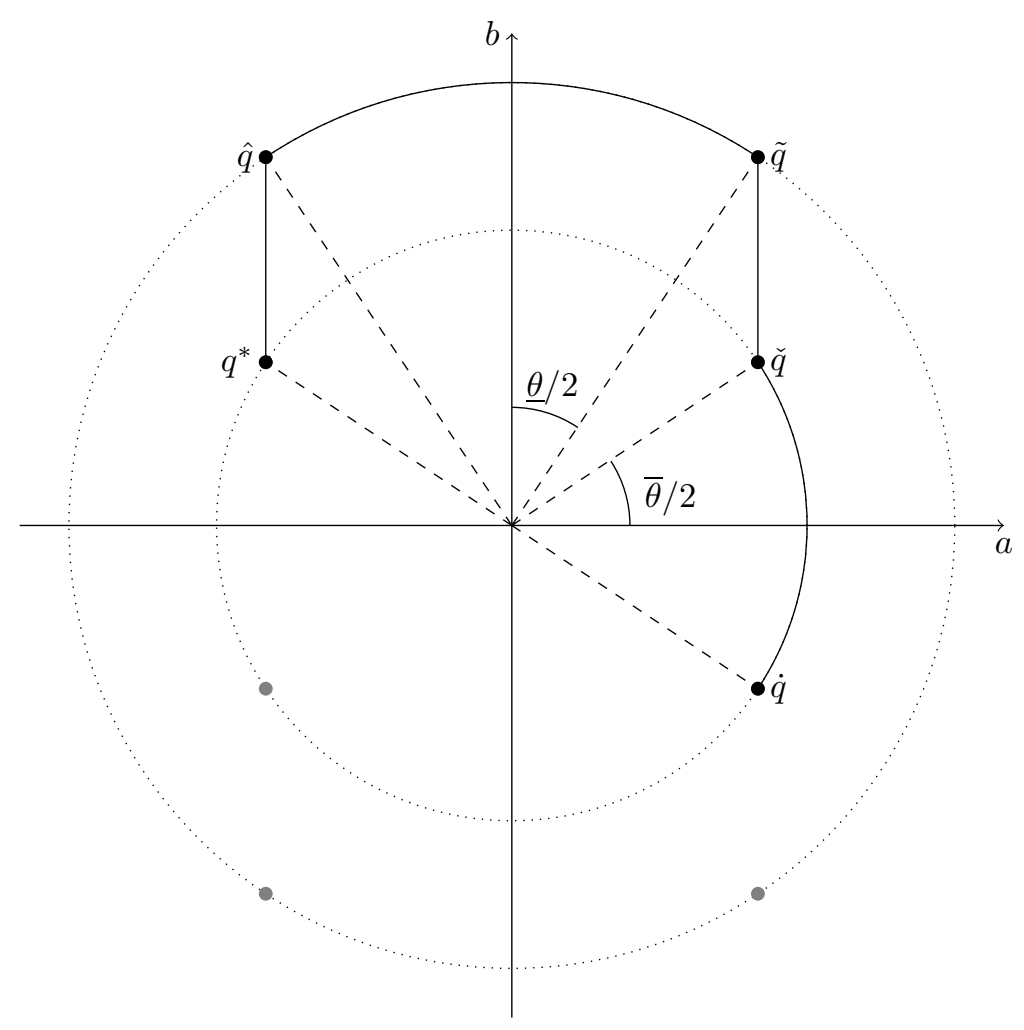

FIG. 7. The states $\dot{q}, \breve{q}, \tilde{q}, \hat{q}, q^{*}$ for $\mathbf{q} \in \Sigma_{1}$.

For the proof of Theorem 9 we use the following lemma.

Lemma 10. Let $q=(a, b), J \neq \pm 1$. If $\|q\|=\left\|q^{\prime}\right\|$ and $\|D q\|=\left\|D q^{\prime}\right\|$, then $q^{\prime}=( \pm a, \pm b)$.

This lemma follows immediately from

$$
a^{2}+b^{2}=\left(a^{\prime}\right)^{2}+\left(b^{\prime}\right)^{2} \text { and } a^{2}+J^{2} b^{2}=\left(a^{\prime}\right)^{2}+J^{2}\left(b^{\prime}\right)^{2},
$$

which are, in turn, direct consequences of (61).

We can now prove the theorem.

Proof of Theorem 9. Assume $M q=-q$ for $q \in \operatorname{Span}\{\dot{q}=(\dot{a}, \dot{b})\}$, where $\dot{q}$ is a representative of the subspace satisfying $\|\dot{q}\|=1$ and $-\pi / 2 \leq \dot{\mu}<\pi / 2$.

First, we prove that $\dot{q}$ must have a nonpositive slope, $\dot{b} / \dot{a} \leq 0$. Assume for contradiction that $\dot{b} / \dot{a}>0$, so that $\dot{q}$ lies in the first quadrant. Then $q^{*}=-\dot{q}$ lies in the third quadrant, and $\hat{q}=D q^{*}$ must also lie in the third quadrant on a circle of radius $\alpha \equiv\|\hat{q}\|>1$ because $J>1$; cf. Figure 8 . Then $\tilde{q}=R(-\underline{\theta}) \hat{q}$ must now lie on the circle of radius $\alpha$, and $\check{q}=D^{-1} \tilde{q}$ lies on the circle of radius 1 . But by Lemma 10 , the only such point on the circle of radius $\alpha$ that lies within an angle of $\pi$ from $\hat{q}$ (and is mapped by $D$ to a point on the circle of radius 1 ) is the point on the circle of radius $\alpha$ directly above the point $\hat{q}$; cf. Figure 8 . Now $\check{q}=D^{-1} \tilde{q}=R(\bar{\theta})$ must thus lie on the intersection of the vertical line through $\check{q}$ and the unit circle. The only way this can happen is if $\tilde{q}, \hat{q}, \tilde{q}$, and $\check{q}$ all lie on the same vertical line, passing through the circles $\|q\|=\alpha$ and $\|q\|=1$; cf. Figure 8 .

That is, $\tilde{q}$ is the only point on the circle of radius $\alpha$ within an angle of $\pi$ from $\hat{q}$ 


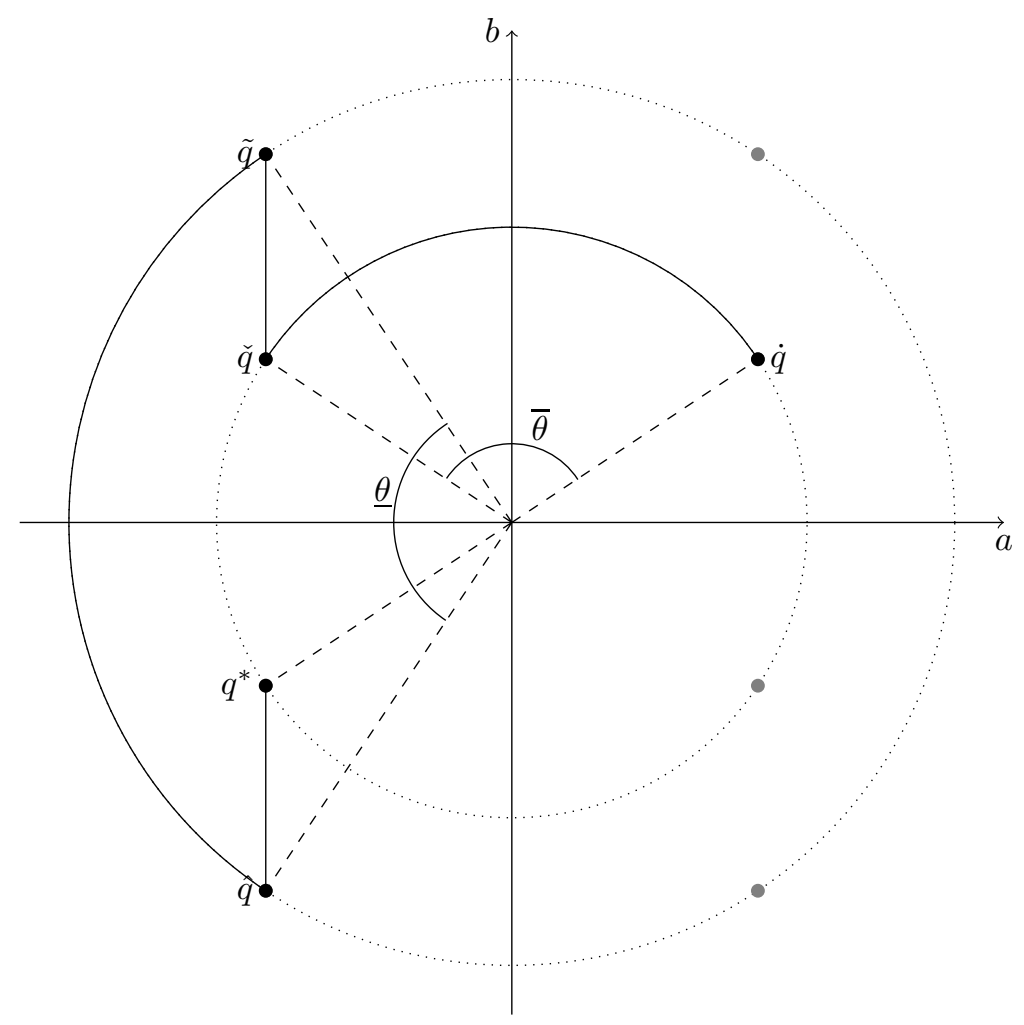

FIG. 8. The states with $\dot{\mu}>0$, showing $\underline{\theta}+\bar{\theta}>\pi$.

that is mapped by $D$ to a point on the circle of radius 1 . From these considerations it follows that

$$
\begin{aligned}
q^{*} & =(-\dot{a},-\dot{b}), \\
\hat{q} & =(-\dot{a},-J \dot{b}), \\
\tilde{q} & =(-\dot{a}, J \dot{b}), \\
\check{q} & =(-\dot{a}, \dot{b}) .
\end{aligned}
$$

From $\check{q}=R(\bar{\theta}) \dot{q}$, it follows that $\dot{\mu}=\pi / 2-\bar{\theta} / 2$ and $\check{\mu}=\pi / 2+\bar{\theta} / 2$, and it follows from $\hat{q}=R(\underline{\theta}) \tilde{q}$ that $\tilde{\mu}=\pi-\bar{\theta} / 2$ and $\hat{\mu}=\pi+\bar{\theta} / 2$; cf. Figure 8 . But it is easily seen from Figure 8 that this configuration of angles implies that $\bar{\theta}+\underline{\theta}>\pi$, a contradiction. Thus we must have $-\pi / 2 \leq \dot{\mu} \leq 0$.

Consider next the case $-\pi / 2<\dot{\mu}<0$. In this case $\dot{b} / \dot{a}<0$, so that $\dot{q}$ lies in the fourth quadrant. Then $q^{*}=-\dot{q}$ must lie in the second quadrant, and $\hat{q}=D q^{*}$ must also lie in the second quadrant on a circle of radius $\alpha=\|\hat{q}\|>1$ because $J>1$. Then $\tilde{q}=R(-\underline{\theta}) \hat{q}$ must lie on the circle of radius $\alpha$, and $\check{q}=D^{-1} \tilde{q}$ then lies on the circle of radius 1. But by Lemma 10, the only such point on the circle of radius $\alpha$ that lies within an angle of $\pi$ from $\hat{q}$ and is mapped by $D$ to a point on the circle of radius 1 is the point on the circle of radius $\alpha$ directly to the right of and on the same horizontal line as the point $\hat{q}$; cf. Figure 7 . Now $\check{q}=D^{-1} \tilde{q}=R(\bar{\theta}) \dot{q}$ must thus lie on the intersection of the vertical line through $\check{q}$ and the unit circle. The only way this 
can happen is if $\dot{q}, \breve{q}$, and $\tilde{q}$ all lie on the same vertical line, with $\|q\|=\alpha$ and $\|q\|=1$; cf. Figure 7. From these considerations it follows that

$$
\begin{aligned}
q^{*} & =(-\dot{a},-\dot{b}), \\
\hat{q} & =(-\dot{a},-J \dot{b}), \\
\tilde{q} & =(\dot{a},-J \dot{b}), \\
\check{q} & =(\dot{a},-\dot{b}),
\end{aligned}
$$

with $\dot{a}>0, \dot{b}<0$. It now follows from $\check{q}=R(\bar{\theta}) \dot{q}$ that $\dot{\mu}=-\bar{\theta} / 2$ and $\check{\mu}=\bar{\theta} / 2$, and it follows from $\hat{q}=R(\underline{\theta}) \tilde{q}$ that $\tilde{\mu}=\pi / 2-\bar{\theta} / 2$ and $\hat{\mu}=\pi / 2+\bar{\theta} / 2$; cf. Figure 7. It is easily seen from Figure 7 that this configuration of angles satisfies the condition $\bar{\theta}+\underline{\theta}<\pi$ and is the unique solution. This verifies (77)-(80). Similar arguments show that the limit cases $\dot{\mu}=0,-\pi / 2$ correspond to $\bar{\theta}=0, \underline{\theta}=\pi$ and $\underline{\theta}=0, \bar{\theta}=\pi$, respectively, both cases that have been ruled out.

Finally, for (75) we have both

$$
\tilde{q}=(\cos (\bar{\theta} / 2), J \sin (\bar{\theta} / 2))
$$

and

$$
\tilde{q}=\alpha(\cos (\pi / 2-\underline{\theta} / 2), \sin (\pi / 2-\underline{\theta} / 2))
$$

where $\alpha=\|\tilde{q}\|$, so that

$$
\alpha^{2}=\cos ^{2}(\bar{\theta} / 2)+J^{2} \sin ^{2}(\bar{\theta} / 2)
$$

Thus comparing first components of (81) and (82), we get

$$
\cos ^{2}(\bar{\theta} / 2)=\left(\cos ^{2}(\bar{\theta} / 2)+J^{2} \sin ^{2}(\bar{\theta} / 2)\right) \cos ^{2}(\pi / 2-\underline{\theta} / 2)
$$

and solving for $J$ leads directly to (75).

4.2. Real valued solutions. The solution $V_{1}(t) \in \Sigma_{1}$ of $(47)$ corresponding to $\mathbf{q}$ is

$$
V_{1}(t)=\mathcal{T}_{1} \mathbf{q}=\left[\begin{array}{c}
\cos (\bar{\theta} / 2) \\
i \sin (\bar{\theta} / 2)
\end{array}\right] e^{i t}
$$

which is never real. To obtain a real solution of (47), we must add the complex conjugate $\overline{V_{1}(t)} \in \Sigma_{-1}$. Now observe that having found $V_{1}$ as a solution of (72), we obtain the solution of (73) by changing the signs of $\bar{\theta}$ and $\underline{\theta}, J$ being invariant under this change of sign. That is,

$$
q_{-1}=\left[\begin{array}{c}
\cos (-\bar{\theta} / 2) \\
-\sin (-\bar{\theta} / 2)
\end{array}\right]=\left[\begin{array}{c}
\cos (\bar{\theta} / 2) \\
\sin (\bar{\theta} / 2)
\end{array}\right]
$$

so that by $(56)$,

$$
V_{-1}(t)=\mathcal{T}_{-1} q_{-1}=\left[\begin{array}{c}
\cos (\bar{\theta} / 2) \\
-i \sin (\bar{\theta} / 2)
\end{array}\right] e^{-i t}
$$

Thus, although there are no real solutions in either $\Sigma_{1}$ or $\Sigma_{-1}$, we see from (83) and (84) that the respective solutions are complex conjugates,

$$
V_{-1}(t)=\overline{V_{1}(t)}
$$


so (54) applies and we obtain the two independent real solutions

$$
V_{a}(t)=\left[\begin{array}{c}
\cos (\bar{\theta} / 2) \cos (t) \\
-\sin (\bar{\theta} / 2) \sin (t)
\end{array}\right]=\frac{1}{2}\left[V_{1}(t)+V_{-1}(t)\right]
$$

and

$$
V_{b}(t)=\left[\begin{array}{c}
\cos (\bar{\theta} / 2) \sin (t) \\
\sin (\bar{\theta} / 2) \cos (t)
\end{array}\right]=\frac{1}{2 i}\left[V_{1}(t)-V_{-1}(t)\right],
$$

which differ by a quarter-period phase shift, $V_{b}(t)=V_{a}\left(t+\frac{\pi}{2}\right)$.

More generally, let $\Sigma^{*}$ denote the set of $V(t) \in \Sigma$ such that $V(t)$ is real. By (51), (53), and (54), $V(t) \in \Sigma^{*}$ if and only if $\overline{V_{-n}}=V_{n}$, and

$$
\overline{V_{-n}} e^{-i n t}+V_{n} e^{i n t}=\left[\begin{array}{c}
\operatorname{Re}\left\{a_{n}\right\} \\
\operatorname{Im}\left\{b_{n}\right\}
\end{array}\right] \cos n t+\left[\begin{array}{c}
\operatorname{Im}\left\{a_{n}\right\} \\
\operatorname{Re}\left\{b_{n}\right\}
\end{array}\right] \sin n t .
$$

Now $\Sigma^{*}$ is the orthogonal direct sum

$$
\Sigma^{*}=\Delta \oplus \Delta^{\perp}
$$

of series even/odd and odd/even in $w / v$, respectively. That is,

$$
\Delta=\oplus_{n=0}^{+\infty} \Delta_{n}
$$

and

$$
\Delta^{\perp}=\oplus_{n=0}^{+\infty} \Delta_{n}^{\perp}
$$

where

$$
\Delta_{n}=\left\{V(t)=\left[\begin{array}{c}
a_{n} \cos n t \\
b_{n} \sin n t
\end{array}\right]: a_{n}, b_{n} \in \mathbb{R}\right\}
$$

and

$$
\Delta_{n}^{\perp}=\left\{V(t)=\left[\begin{array}{c}
c_{n} \sin n t \\
d_{n} \cos n t
\end{array}\right]: c_{n}, d_{n} \in \mathbb{R}\right\}
$$

The following corollary is now a direct consequence of Theorem 9.

Corollary 11. The functions $V_{a}(t) \in \Delta_{1}$ and $V_{b}(t) \in \Delta_{1}^{\perp}$ are, to within a factor, the only two real solutions of (47) in $\Delta_{1}$ and $\Delta_{1}^{\perp}$, respectively.

We now refer back to Figures 2 and 3, which are now exact descriptions of the solutions $V_{a}(t)$ of the eigenvalue problem (47) and the corresponding solutions of the PDE (46). That is, $V_{a}(t)$ as a curve is precisely the $\dot{U}$ ellipse, and the other ellipses are the curves corresponding to the linear evolutions and jumps of $V_{a}$. Moreover, in the nondimensional version of Figure 2, all characteristics would have slope \pm 1 , and so the characteristic diagram would be exact. We note that Figure 2 has an extra symmetry in that the $\max / \min$ characteristics return. A characteristic diagram in which this does not happen is given in Figure 9.

Since the real solutions $V_{a}$ and $V_{b}$ differ by a phase shift, and the PDEs (46) have no explicit $t$-dependence, these are essentially the same solution. That is, the PDEs have a phase translation symmetry, and any solution generates another by a 
fixed phase shift. We remove the effects of this phase symmetry by observing that the nonlinear PDEs (29) are invariant under the mapping

$$
w(y, t) \rightarrow w(y,-t) \quad \text { and } \quad v(y, t) \rightarrow-v(y,-t),
$$

which also preserves the space $\Delta=\oplus \Delta_{n}$.

LEMMA 12. If $V(t)=(w(t), v(t)) \in \Delta$ is $2 \pi$-periodic, sufficiently smooth, and sufficiently small, then both $\mathcal{M}[V(\cdot)](t)$ and $\mathcal{N}[V(\cdot)](t)$ are well-defined smooth functions, and

$$
\mathcal{M}[V(\cdot)] \in \Delta \quad \text { and } \quad \mathcal{N}[V(\cdot)] \in \Delta .
$$

Proof. By the regularity of smooth solutions for the $2 \times 2$ systems of conservation laws (29) and (46), together with the fact that $\mathcal{J}$ and $\mathcal{S}$ are linear operators, it follows that $\mathcal{M}[V(\cdot)](t)$ and $\mathcal{N}[V(\cdot)](t)$ are well-defined functions in $C^{k}$ or $H^{s}$ for $V(t)$ sufficiently small in $C^{k}$ or $H^{s}$, respectively, $s, k \geq 2$, (cf. [17, Theorem 2.2, page $46])$. Thus to verify (86), it suffices to show that for such $V(t)=V(0, t)$ in the domain of $\mathcal{M}$ and $\mathcal{N}$, if $V(y, t)=(w(y, t), v(y, t))$ is even in $w$ and odd in $v$ at $y=0$, then it is even in $w$ and odd in $v$ for all $0 \leq y \leq \bar{\theta}+\underline{\theta}$, where

$$
V(y, t)= \begin{cases}\mathcal{E}(y) V(\cdot), & 0<y<\bar{\theta} \\ \mathcal{E}(y-\bar{\theta}) \mathcal{J E}(\bar{\theta}) V(\cdot), & \bar{\theta}<y<\bar{\theta}+\underline{\theta} \\ \mathcal{S} \mathcal{J}^{-1} \mathcal{E}(\underline{\theta}) \mathcal{J} \mathcal{E}(\bar{\theta}) V(\cdot), & y=\bar{\theta}+\underline{\theta}\end{cases}
$$

But the property even in $w$ odd in $v$ is clearly preserved by operators $\mathcal{J}, \mathcal{J}^{-1}$, and $\mathcal{S}$, so it suffices to show that even in $w$ odd in $v$ is preserved by the nonlinear evolution $\mathcal{E}$ of system (29). Since solutions of (29) are invariant under the mapping $w(y, t) \rightarrow$ $w(y,-t)$ and $v(y, t) \rightarrow-v(y,-t)$, we can extend a solution $V(y, t)$ from $t \geq 0$ to $t \leq 0$ by the reflection

$$
V(y,-t)=(w(t),-v(t))
$$

By the uniqueness of continuous solutions for smooth initial data, we need only show that the matched solution is continuous at $t=0$ to conclude it is unique, and hence even in $w$ odd in $v$ by construction. Continuity in $w$ at $t=0$ is guaranteed by $w(t)=w(-t)$. For continuity of $v$ at $t=0$, we need to show that $v(y, 0)=0$ for all $0 \leq y \leq \bar{\theta}+\underline{\theta}$. For this the only real issue is to show that the nonlinear evolution (29) preserves $v(y, 0)=0$. To verify this, transform (29) to Riemann invariant coordinates $r=v-w, s=v+w$, leading to the equivalent system

$$
\begin{aligned}
& r_{y}-\sigma r_{t}=0, \\
& s_{y}+\sigma s_{t}=0 .
\end{aligned}
$$

It follows that $r, s$ are constant along characteristics $d t / d y=-\sigma, d t / d y=\sigma$, respectively. Tracing the characteristics back from point $(w(y, 0), v(y, 0))$ to points $(w(0, \pm t), v(0, \pm t))$ and using even in $w$ odd in $v$ at $y=0$ gives

$$
\begin{aligned}
& v(y, 0)+w(y, 0)=-v(0, t)+w(0, t), \\
& v(y, 0)-w(y, 0)=v(0, t)-w(0, t),
\end{aligned}
$$

which upon adding leads to $v(y, 0)=0$ as claimed. 
We now clarify the representation of the linear operator $\mathcal{M}-\mathcal{I}$ in the real Hilbert space $\Delta$. To set notation, as the real counterpart of (56), for $n \geq 0$ we define the real representation $\mathcal{T}_{n}^{*}: \mathbb{R}^{2} \rightarrow \Delta_{n}$ by

$$
\begin{aligned}
\mathcal{T}_{n}^{*}\left[\begin{array}{l}
a \\
b
\end{array}\right] & =\frac{1}{2}\left\{\mathcal{T}_{n}\left[\begin{array}{l}
a \\
b
\end{array}\right]+\mathcal{T}_{-n} H\left[\begin{array}{l}
a \\
b
\end{array}\right]\right\} \\
& \equiv\left[\begin{array}{c}
a \cos (n t) \\
b \sin (n t)
\end{array}\right]
\end{aligned}
$$

where $H$ denotes the matrix

$$
H=\left(\begin{array}{cc}
1 & 0 \\
0 & -1
\end{array}\right)
$$

We now have the following real analogue of Theorem 6 .

Lemma 13. The linear operator $\mathcal{M}$ restricted to $\Delta$ has the orthogonal representation

$$
\mathcal{M}=\oplus_{n=0}^{\infty} \mathcal{M}_{n}
$$

where

$$
\mathcal{M}_{n}: \Delta_{n} \rightarrow \Delta_{n}
$$

is given by

$$
\mathcal{M}_{n}\left(\mathcal{T}_{n}^{*}\left[\begin{array}{l}
a_{n} \\
b_{n}
\end{array}\right]\right)=\mathcal{T}_{n}^{*}\left(M_{n}\left[\begin{array}{l}
a_{n} \\
b_{n}
\end{array}\right]\right)
$$

where, as in (63), (64), and (65), $M_{n}$ is the $2 \times 2$ real matrix

$$
M_{n}=(-1)^{n} D^{-1} R(n \underline{\theta}) D R(n \bar{\theta})=(-1)^{n} M(n \bar{\theta}, n \underline{\theta}, J) .
$$

Proof. First, note that $H$ commutes with $D$, and

$$
R(-\theta) H=H R(\theta)
$$

for all angles $\theta$, which yields the matrix identity

$$
M_{-n} H=H M_{n} .
$$

Then for $n \geq 0$ and any real $a_{n}, b_{n}$, by (88) and Theorem 6 , we have

$$
\begin{aligned}
\mathcal{M}\left(\mathcal{T}_{n}^{*}\left[\begin{array}{l}
a_{n} \\
b_{n}
\end{array}\right]\right) & =\mathcal{M}\left(\frac{1}{2}\left\{\mathcal{T}_{n}\left[\begin{array}{l}
a \\
b
\end{array}\right]+\mathcal{T}_{-n} H\left[\begin{array}{l}
a \\
b
\end{array}\right]\right\}\right) \\
& =\frac{1}{2}\left\{\mathcal{T}_{n} M_{n}\left[\begin{array}{l}
a \\
b
\end{array}\right]+\mathcal{T}_{-n} M_{-n} H\left[\begin{array}{l}
a \\
b
\end{array}\right]\right\} \\
& =\frac{1}{2}\left\{\mathcal{T}_{n} M_{n}\left[\begin{array}{l}
a \\
b
\end{array}\right]+\mathcal{T}_{-n} H M_{n}\left[\begin{array}{l}
a \\
b
\end{array}\right]\right\} \\
& =\mathcal{T}_{n}^{*}\left(M_{n}\left[\begin{array}{l}
a \\
b
\end{array}\right]\right)
\end{aligned}
$$

as required. 
We summarize the results in the following theorem.

THEOREM 14. The operator $\mathcal{M}-\mathcal{I}: \Delta \rightarrow \Delta$ has the decomposition

$$
\mathcal{M}-\mathcal{I}=\oplus_{n=0}^{\infty}\left(\mathcal{M}_{n}-\mathcal{I}\right),
$$

where $\mathcal{M}_{n}-\mathcal{I}: \Delta_{n} \rightarrow \Delta_{n}$ acts by

$$
\left(\mathcal{M}_{n}-\mathcal{I}\right)\left(\mathcal{T}_{n}^{*} \mathbf{p}_{n}\right)=\mathcal{T}_{n}^{*}\left[\left(M_{n}-I\right) \mathbf{p}_{n}\right] .
$$

Moreover, if

$$
J>1, \quad \bar{\theta}>0, \quad \underline{\theta}>0,
$$

and

$$
\bar{\theta}+\underline{\theta}<\pi,
$$

then $V(t)$ is a solution of $(\mathcal{M}-\mathcal{I})[V]=0$ if and only if

$$
J=\cot (\bar{\theta} / 2) \cot (\underline{\theta} / 2) ;
$$

$c f .(75)$, and

$$
V(t)=V_{a}(t) \equiv\left[\begin{array}{c}
\cos (\bar{\theta} / 2) \cos (t) \\
-\sin (\bar{\theta} / 2) \sin (t)
\end{array}\right] ;
$$

cf. (85).

From here on we restrict ourselves to the real $L^{2}$ space $\Delta$ of functions even in $w$, odd in $v$, and to the linearized operator

$$
\mathcal{M}-\mathcal{I}: \Delta \rightarrow \Delta,
$$

under the assumptions (89), (90). Our purpose in the next two sections is to analyze the spectrum of $\mathcal{M}-\mathcal{I}$ with the aim of finding conditions on $(\bar{\theta}, \underline{\theta})$ sufficient to isolate the solution $V_{a}(t)$ in the kernel of $\mathcal{M}-\mathcal{I}$, the condition required for a LyapunovSchmidt decomposition.

5. Wave structure of the linearized solutions. In this section we reconstruct and discuss the linear periodic solution associated with $V_{a}(t)$ defined in (91) of Theorem 14. Since $(\mathcal{M}-\mathcal{I})\left[V_{a}\right](\cdot)=0$, we can use (48) and (43), together with the fact that $\mathcal{L}$ is the linearization of $\mathcal{E}$ about the constant state $(1,0)$, to obtain the linearized solution $V_{a}(y, t)=\left(w_{a}(y, t), v_{a}(y, t)\right)$ in dimensionless state variables $(w, v)$, dimensionless space variable $y$, and dimensionless periods $(\bar{\theta}, \underline{\theta})$; cf. (25), (26), (45), (87), and Theorem 2:

$$
V_{a}(y, \cdot) \equiv \mathcal{M}(y)\left[V_{a}(\cdot)\right] \equiv \begin{cases}\mathcal{L}(y)\left[V_{a}(\cdot)\right], & 0 \leq y<\bar{\theta} \\ \mathcal{L}(y-\bar{\theta}) \mathcal{J} \mathcal{L}(\bar{\theta})\left[V_{a}(\cdot)\right], & 0<y-\bar{\theta}<\underline{\theta}, \\ \mathcal{S} \mathcal{J}^{-1} \mathcal{L}(\underline{\theta}) \mathcal{J} \mathcal{L}(\bar{\theta})\left[V_{a}(\cdot)\right], & y=\bar{\theta}+\underline{\theta} .\end{cases}
$$

The condition $(\mathcal{M}-\mathcal{I})\left[V_{a}\right](\cdot)=0$ implies that $V_{a}(y, t)$ is $2 \pi$-periodic in time and $(\bar{\theta}+\underline{\theta})$-periodic in space, after a $\pi$-time translation. Since $\mathcal{S}^{2}=\mathcal{I}$, it follows that $V_{a}(y, t)$ is exactly $(\bar{\theta}+\underline{\theta})$-periodic, but the rectangle $[0, \bar{\theta}+\underline{\theta}) \times[0,2 \pi)$ is a minimum 
spacetime-periodic tile with a period translation vector $\mathbf{X}=(\bar{\theta}+\underline{\theta}) \mathbf{e}_{y}+\pi \mathbf{e}_{t}$; that is,

$$
V_{a}(y, t)=V_{a}((y, t)+\mathbf{X}) .
$$

To obtain exact formulas for $V_{a}(y, t)$, recall that by $(45)$, the linear evolution $\mathcal{L}(y)[V(\cdot)]$ is given by evolution through "time" $y$ by the linear system (46),

$$
\begin{aligned}
& w_{y}+v_{t}=0, \\
& v_{y}+w_{t}=0,
\end{aligned}
$$

starting with initial data $V(\cdot)$. Now for $0 \leq y<\bar{\theta}$, we have (cf. Theorem 9 and (88))

$$
V_{a}(y, t)=\left[\begin{array}{c}
w_{a}(y, t) \\
v_{a}(y, t)
\end{array}\right]=\mathcal{L}(y)\left[\begin{array}{c}
w_{a}(0, t) \\
v_{a}(0, t)
\end{array}\right]=\mathcal{L}(y) \mathcal{T}_{1}^{*}[q]=\mathcal{T}_{1}^{*} R(y) q,
$$

where by (77)

$$
q=\left[\begin{array}{c}
\cos (\bar{\theta} / 2) \\
-\sin (\bar{\theta} / 2)
\end{array}\right]=R\left(-\frac{\bar{\theta}}{2}\right)\left[\begin{array}{l}
1 \\
0
\end{array}\right]
$$

so that

$$
R(y) q=R\left(y-\frac{\bar{\theta}}{2}\right)\left[\begin{array}{l}
1 \\
0
\end{array}\right]=[\cos (y-\bar{\theta} / 2) \sin (y-\bar{\theta} / 2)]
$$

Thus

$$
V_{a}(y, t)=\left[\begin{array}{c}
\cos (y-\bar{\theta} / 2) \cos t \\
\sin (y-\bar{\theta} / 2) \sin t
\end{array}\right], \quad 0 \leq y<\bar{\theta} .
$$

Similarly, for $\bar{\theta} \leq y<\bar{\theta}+\underline{\theta}$ we have (cf. (78))

$$
V_{a}(y, t)=\mathcal{L}(y-\bar{\theta}) \mathcal{T}_{1}^{*}[\tilde{q}]
$$

where by (77)

$$
\tilde{q}=\alpha R\left(\frac{-\underline{\theta}}{2}\right)\left[\begin{array}{l}
0 \\
1
\end{array}\right],
$$

with

$$
\alpha=\|\tilde{q}\|=\frac{\cos (\bar{\theta} / 2)}{\sin (\underline{\theta} / 2)},
$$

and we have used (75). Thus for $\bar{\theta} \leq y<\bar{\theta}+\underline{\theta}$,

$$
\begin{aligned}
V_{a}(y, t) & =\alpha \mathcal{T}_{1}^{*} R(y-\bar{\theta}) R\left(\frac{-\underline{\theta}}{2}\right)\left[\begin{array}{l}
0 \\
1
\end{array}\right] \\
& =\alpha \mathcal{T}_{1}^{*} R\left(y-\bar{\theta}-\frac{\theta}{2}\right)\left[\begin{array}{l}
0 \\
1
\end{array}\right],
\end{aligned}
$$


which yields

$$
V_{a}(y, t)=\frac{\cos (\bar{\theta} / 2)}{\sin (\underline{\theta} / 2)}\left[\begin{array}{c}
-\sin \left(y-\bar{\theta}-\frac{\theta}{2}\right) \cos t \\
\sin \left(y-\bar{\theta}-\frac{\theta}{\overline{2}}\right) \sin t
\end{array}\right], \quad \bar{\theta} \leq y<\bar{\theta}+\underline{\theta} .
$$

Letting

$$
V_{\Theta}(y, t)=\sin (\underline{\theta} / 2) V_{a}(y, t),
$$

we obtain from (93) and (94) a closed form expression for a linearized periodic solution of the compressible Euler equations with spatial periods $\bar{\theta}, \underline{\theta}$. We summarize these results in the following theorem.

THEOREM 15. For each choice of positive periods $0<\bar{\theta}+\underline{\theta}<\pi$, the following closed form expression defines a linearized periodic solution $V(y, t)$ of the compressible Euler equations in dimensionless variables $w, v, y, \theta$ :

$$
V_{\Theta}(y, t)= \begin{cases}\sin (\underline{\theta} / 2)\left[\begin{array}{c}
\cos (y-\bar{\theta} / 2) \cos t \\
\sin (y-\bar{\theta} / 2) \sin t
\end{array}\right], & 0 \leq y<\bar{\theta}, \\
\cos (\bar{\theta} / 2)\left[\begin{array}{c}
-\sin \left(y-\bar{\theta}-\frac{\theta}{2}\right) \cos t \\
\sin \left(y-\bar{\theta}-\frac{\theta}{2}\right) \sin t
\end{array}\right], \quad \bar{\theta} \leq y<\bar{\theta}+\underline{\theta} .\end{cases}
$$

Here, the $(y, t)$-region $[0, \bar{\theta}+\underline{\theta}) \times[0,2 \pi)$ defines one spacetime period, and the entire solution is obtained by mapping the solution in the period $[0, \bar{\theta}+\underline{\theta}) \times[0,2 \pi)$ to the $(y, t)$-plane via translation (92). In particular, the Rankine-Hugoniot jump conditions hold at the entropy jumps $y=\bar{\theta}$ and $y=\bar{\theta}+\underline{\theta}$, and the resulting solution has the property that nearby nonlinear solutions formally balance compression and rarefaction along characteristics in the sense of [25].

Using the transformations (10) and (23)-(33) we obtain the following theorem regarding linearized periodic solutions of the dimensional compressible Euler equations.

THEOREM 16. For each base state $(\bar{\tau}, \bar{v}, \bar{S})$ and adiabatic constant $\gamma$, formula (95) for $V_{\Theta}$ determines a two parameter family of linearized periodic solutions of the compressible Euler equations determined by $\Theta=(\bar{\theta}, \underline{\theta}), 0<\bar{\theta}+\underline{\theta}$.

Proof. The states $(\bar{\tau}, \bar{u}, \bar{S})$ uniquely determine corresponding state $(\bar{z}, \bar{u}, \bar{m})$ through transformations (10). This in turn gives the upper and lower bar constant states associated with dimensional solutions in the following order: equation (12) gives

$$
\bar{c}=K_{c} \bar{m} \bar{z}^{\frac{\gamma+1}{\gamma-1}} .
$$

Equation (12) together with $\bar{\theta}$ gives

$$
\bar{x}=\bar{c} \bar{\theta} .
$$

Using (36) and the value $\underline{\theta}$ gives

$$
J=\cot \left(\frac{\bar{\theta}}{2}\right) \cot \left(\frac{\underline{\theta}}{2}\right) \equiv\left(\frac{\bar{m}}{\underline{m}}\right)^{1 / \gamma} .
$$




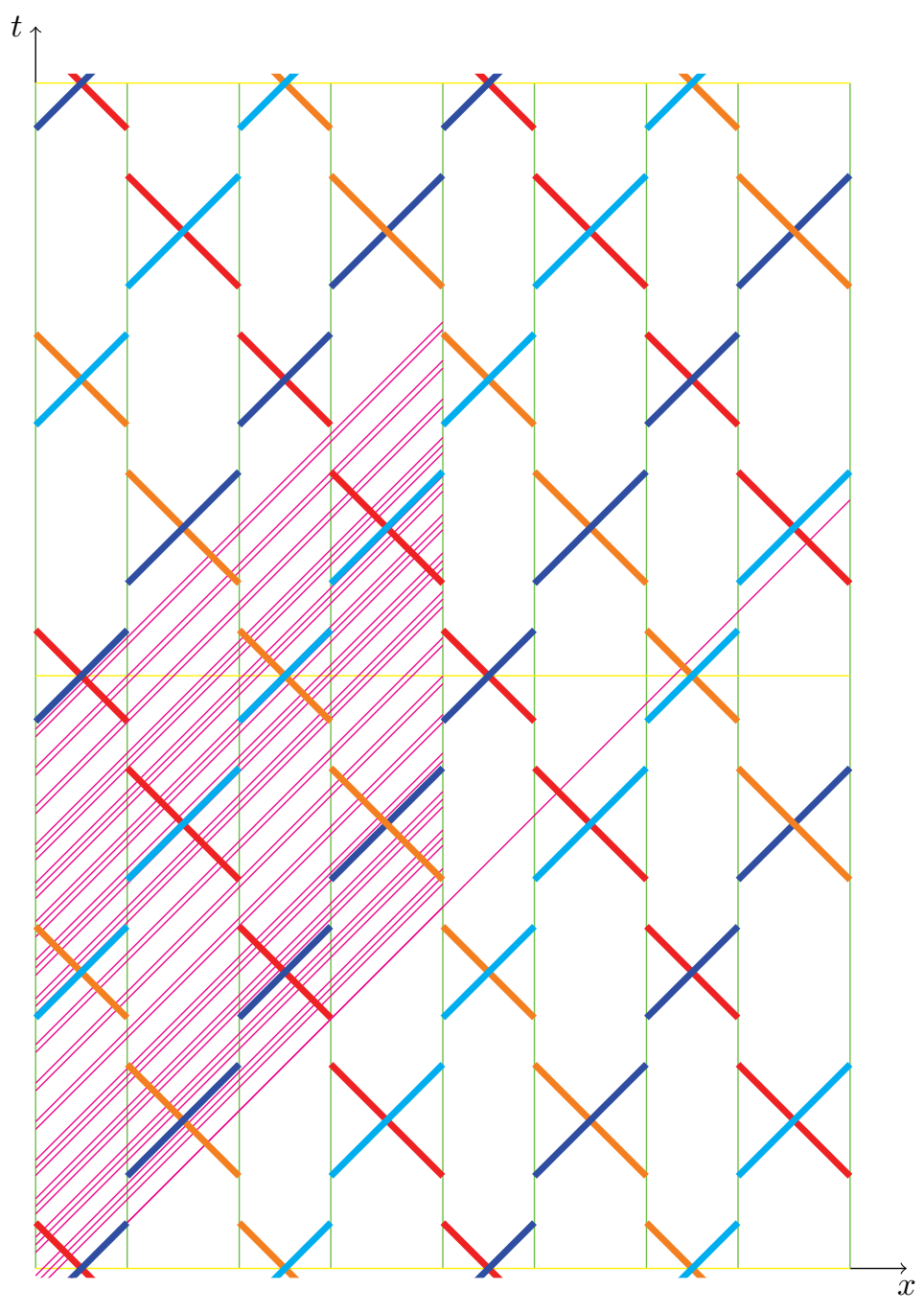

FIG. 9. The dimensionless linearized periodic solution $V_{\Theta}(y, t)$ showing incommensurability.

From these values we can get the remaining values of the dimensional solution via the transformations to dimensionless variables (23)-(33). That is,

$$
\underline{z}=\bar{z}\left(\frac{\bar{m}}{\underline{m}}\right)^{1-1 / \gamma}
$$

and

$$
\underline{c}=K_{c} \underline{m} \underline{z}^{\frac{\gamma+1}{\gamma-1}}
$$

and finally

$$
\underline{x}=\underline{c} \underline{\theta} .
$$

Using these states, we obtain a unique dimensional solution from $V_{\Theta}$ in the region 
$0 \leq y \leq \bar{\theta}$ by the transformations

$$
z=\bar{z} w, \quad v=\frac{u-\bar{u}}{\overline{m z}}, \quad \text { and } \quad x=\bar{c} y,
$$

and in the region $\bar{\theta} \leq y \leq \bar{\theta}+\underline{\theta}$ by the transformations

$$
z=\underline{z} w, \quad v=\frac{u-\underline{u}}{\underline{m} \underline{z}}, \quad \text { and } \quad x=\bar{x}+\bar{c}(y-\bar{\theta}) .
$$

After making the above substitutions into the right-hand side of the formula for $V_{\Theta}$ in (95), we obtain one period of the dimensional periodic linearized solution $U_{\Theta}(x, t)$ defined for $(x, t) \in[0, \bar{x}+\underline{x}) \times[0,2 \pi)$. For example, by construction this solution will satisfy the Rankine-Hugoniot relations at the entropy jumps $x=\bar{x}$ and $x=0, \bar{x}+\underline{x}$, and hence at every entropy jump by periodicity.

The linearized solution $U_{\Theta}(x, t)$ in dimensional variables is depicted in Figures 2 and 3 , in the case $\bar{\theta}=\underline{\theta}=\pi / 4$. Note that the solution is described by an ellipse in $t$ at each fixed $x$, and note that this is the maximally symmetric case in which the sound speed is commensurate with the period speed, and each characteristic returns periodically after traversing eight entropy levels (that is, two spatial periods). The states labeled in the characteristic diagram, Figure 2 in the $(x, t)$-plane, correspond to the labeled states on the ellipses in state space, Figure 3.

In Figure 9, the characteristics of the dimensionless solution $V_{\Theta}$ in $(y, t)$-space are depicted for the case $\bar{\theta}=\pi /(1+\sqrt{5}), \underline{\theta}=\pi / \sqrt{7}$, a case in which the sound speed is incommensurate, i.e., irrationally related to the speed of the period. In this case the characteristics are quasiperiodic, and the balancing of rarefaction and compression along characteristics is achieved by ergodic motion through the period.

6. The eigenvalues of the linearized operators. In this section we state and prove some basic properties of the eigenvalues of the linearized operator $\mathcal{M}-\mathcal{I}: \Delta \rightarrow$ $\Delta$; cf. (48), (70). These properties will be used in the next section to obtain conditions on the periods under which the linearized operator is nonresonant in the sense that the operator is invertible on the orthogonal complement of the 1-mode solution $V_{a}$ that we have constructed in the kernel of the operator; cf. (85). For example, in the nonresonant case, the operator is amenable to a Lyapunov-Schmidt decomposition in bifurcation theory [7].

To start, note that by Theorem 8 the eigenvalues of $M(n \bar{\theta}, n \underline{\theta}, J)$ are

$$
\lambda_{n}^{ \pm}=\beta_{n} \pm \sqrt{\beta_{n}^{2}-1},
$$

where

$$
\beta_{n} \equiv \beta_{n}(\underline{\theta}, \bar{\theta})=\beta(n \underline{\theta}, n \bar{\theta}),
$$

and the function $\beta$ is defined by

$$
\beta\left(\theta_{1}, \theta_{2}\right)=\cos \left(\theta_{1}\right) \cos \left(\theta_{2}\right)-\frac{J^{2}+1}{2 J} \sin \left(\theta_{1}\right) \sin \left(\theta_{2}\right),
$$

and we can assume $0<\theta_{1}, \theta_{2}<2 \pi$. Thus

$$
\beta_{n}=\cos (n \underline{\theta}) \cos (n \bar{\theta})-\frac{J^{2}+1}{2 J} \sin (n \underline{\theta}) \sin (n \bar{\theta}),
$$


where $\underline{\theta}, \bar{\theta}$ and $J \geq 1$ are parameters. In (74) we restricted ourselves to $0 \leq \underline{\theta}+\bar{\theta} \leq \pi$, which we now view as fixed, but because, starting with such $\Theta=(\underline{\theta}, \overline{\bar{\theta}})$, the twodimensional angle $n \Theta=(n \underline{\theta}, n \bar{\theta})$ goes out of this range, we must consider one full period $0 \leq \theta_{1}, \theta_{2} \leq 2 \pi$ of $\beta\left(\theta_{1}, \theta_{2}\right)$, with $J>1$ regarded as fixed. It is convenient to work directly with $\beta_{n}$, rather than $\lambda_{n}^{ \pm}$, and we will make use of the following lemma.

Lemma 17 . Let $\beta \in \mathbb{R}$. Then

$$
\mid \beta-1 \pm \sqrt{\beta^{2}-1 \mid} \geq \frac{\sqrt{|\beta-1|}}{\sqrt{|\beta|+1}}
$$

and

$$
\mid \beta+1 \pm \sqrt{\beta^{2}-1 \mid} \geq \frac{\sqrt{|\beta+1|}}{\sqrt{|\beta|+1}}
$$

Proof. First, note that (100) follows from (99) by substituting $-\beta$ for $\beta$. To verify (99), write

$$
\left|\beta-1 \pm \sqrt{\beta^{2}-1}\right|=|\sqrt{\beta-1} \pm \sqrt{\beta+1}| \sqrt{|\beta-1|},
$$

where we allow complex values of the square root. It suffices to show that

$$
C_{ \pm}(\beta) \equiv|\sqrt{\beta-1} \pm \sqrt{\beta+1}| \geq \frac{1}{\sqrt{|\beta|+1}}
$$

holds for all $\beta \in \mathbb{R}$. But if $1 \leq \beta<\infty$, then we can estimate

$$
\begin{aligned}
C_{ \pm}(\beta) & =|\sqrt{\beta+1} \mp \sqrt{\beta-1}| \geq|\sqrt{\beta+1}-\sqrt{\beta-1}| \\
& =\frac{(\sqrt{\beta+1}-\sqrt{\beta-1})(\sqrt{\beta+1}+\sqrt{\beta-1})}{\sqrt{\beta+1}+\sqrt{\beta-1}} \\
& =\frac{2}{\sqrt{\beta+1}+\sqrt{\beta-1}} \geq \frac{2}{\sqrt{\beta+1}+\sqrt{\beta+1}} \\
& \geq \frac{1}{\sqrt{|\beta|+1}},
\end{aligned}
$$

as claimed. For the case $-\infty<\beta \leq-1$, we have

$$
C_{ \pm}(\beta)=|\sqrt{\beta+1} \mp \sqrt{\beta-1}|,
$$

where $\sqrt{\beta+1}=i \sqrt{|\beta|-1}$ and $\sqrt{\beta-1}=i \sqrt{|\beta|+1}$ are both imaginary. In this case we can estimate

$$
\begin{aligned}
C_{ \pm}(\beta) & =|\sqrt{|\beta|-1} \pm \sqrt{|\beta|+1}| \\
& \geq|\sqrt{|\beta|-1}-\sqrt{|\beta|+1}| \\
& \geq \frac{1}{\sqrt{|\beta|+1}},
\end{aligned}
$$


where in the last line we have applied the result from the previous case. Consider finally the case $-1<\beta<1$. In this case, $\sqrt{\beta+1}>0$ and $\sqrt{\beta-1}=i \sqrt{1-\beta}$, so

$$
\begin{aligned}
|\sqrt{\beta-1} \pm \sqrt{\beta+1}| & =|\sqrt{\beta+1} \pm i \sqrt{1-\beta}| \\
& =|\sqrt{(\beta+1)+(1-\beta)}|=\sqrt{2} \\
& \geq \frac{1}{\sqrt{|\beta|+1}}
\end{aligned}
$$

as claimed, thus establishing (99) and Lemma 17.

To address the resonance problem in the next section, we now define a convenient change of angles that consolidates the functions $\beta_{n} \pm 1$ into a single function. That is, let

$$
\begin{aligned}
& \phi=\frac{\pi-\underline{\theta}+\bar{\theta}}{2}, \\
& \psi=\frac{\pi-\underline{\theta}-\bar{\theta}}{2},
\end{aligned}
$$

so that $(\underline{\theta}, \bar{\theta}) \rightarrow(\phi, \psi)$ defines a global regular invertible map from $\mathbb{R}^{2} \rightarrow \mathbb{R}^{2}$ with inverse

$$
\bar{\theta}=\phi-\psi, \quad \underline{\theta}=\pi-\psi-\phi .
$$

Note that this change of angles transforms our region of interest

$$
\underline{\theta}>0, \quad \bar{\theta}>0, \quad \underline{\theta}+\bar{\theta}<\pi
$$

into the region

$$
0<\psi<\phi<\pi-\psi \text {. }
$$

Define also

$$
Q=\frac{J-1}{J+1},
$$

mapping $J>1$ into $0<Q<1$ with inverse

$$
J=\frac{1+Q}{1-Q} .
$$

The purpose of these changes of variables is evident in the following lemma.

LEMMA 18. Under the change of variables (101)-(102), for each $n \in \mathbb{N}$, we have

$$
1-(-1)^{n} \beta_{n}(\underline{\theta}, \bar{\theta})=\frac{2}{1-Q^{2}} g_{Q}(n \phi, n \psi),
$$

where

$$
g_{Q}(\phi, \psi) \equiv \sin ^{2}(\psi)-Q^{2} \sin ^{2}(\phi)
$$


Proof. We make repeated use of trigonometric identities. First, set

$$
\beta\left(\theta_{1}, \theta_{2}\right)=\cos \left(\theta_{1}\right) \cos \left(\theta_{2}\right)-\frac{1}{2}\left(J+\frac{1}{J}\right) \sin \left(\theta_{1}\right) \sin \left(\theta_{2}\right),
$$

so that $\beta_{n}=\beta(n \underline{\theta}, n \bar{\theta})$, and use the abbreviations

$$
c_{1}=\cos \left(\theta_{1} / 2\right), \quad s_{1}=\sin \left(\theta_{1} / 2\right), \quad c_{2}=\cos \left(\theta_{2} / 2\right), \quad \text { and } \quad s_{2}=\sin \left(\theta_{2} / 2\right) .
$$

Then using the double-angle formulas, we calculate

$$
\begin{aligned}
1+\beta\left(\theta_{1}, \theta_{2}\right)= & 1+\cos \theta_{1} \cos \theta_{2}-\frac{J^{2}+1}{2 J} \sin \theta_{1} \sin \theta_{2} \\
= & \left(c_{1}{ }^{2}+s_{1}{ }^{2}\right)\left(c_{2}{ }^{2}+s_{2}{ }^{2}\right)+\left(c_{1}{ }^{2}-s_{1}{ }^{2}\right)\left(c_{2}{ }^{2}-s_{2}{ }^{2}\right) \\
& \quad-\frac{J^{2}+1}{2 J}\left(2 s_{1} c_{1}\right)\left(2 s_{2} c_{2}\right) \\
= & 2\left(c_{1} c_{2}-J s_{1} s_{2}\right)\left(c_{1} c_{2}-s_{1} s_{2} / J\right) .
\end{aligned}
$$

Thus, writing

$$
c_{+}=\cos \left(\frac{\theta_{1}}{2}+\frac{\theta_{2}}{2}\right) \quad \text { and } \quad c_{-}=\cos \left(\frac{\theta_{1}}{2}-\frac{\theta_{2}}{2}\right),
$$

so that $c_{1} c_{2}=\left(c_{-}+c_{+}\right) / 2$ and $s_{1} s_{2}=\left(c_{-}-c_{+}\right) / 2$, and substituting in, we get, after rearranging,

$$
1+\beta\left(\theta_{1}, \theta_{2}\right)=\frac{(J+1)^{2}}{2 J}\left(c_{+}-\frac{J-1}{J+1} c_{-}\right)\left(c_{+}+\frac{J-1}{J+1} c_{-}\right) .
$$

Similarly, writing

$$
s_{+}=\sin \left(\frac{\theta_{1}}{2}+\frac{\theta_{2}}{2}\right) \quad \text { and } \quad s_{-}=\sin \left(\frac{\theta_{1}}{2}-\frac{\theta_{2}}{2}\right),
$$

we calculate

$$
\begin{aligned}
1-\beta\left(\theta_{1}, \theta_{2}\right) & =2\left(s_{1} c_{2}+J c_{1} s_{2}\right)\left(s_{1} c_{2}+c_{1} s_{2} / J\right) \\
& =\frac{(J+1)^{2}}{2 J}\left(s_{+}-\frac{J-1}{J+1} s_{-}\right)\left(s_{+}+\frac{J-1}{J+1} s_{-}\right) .
\end{aligned}
$$

Now, making the substitution (102), (103) and using (104), (106) becomes

$$
1-\beta\left(\theta_{1}, \theta_{2}\right)=\frac{2}{1-Q^{2}} g_{Q}\left(\frac{\theta_{1}}{2}-\frac{\theta_{2}}{2}, \frac{\theta_{1}}{2}+\frac{\theta_{2}}{2}\right),
$$

and using the identity $\cos (x)=-\sin (x-\pi / 2),(105)$ becomes

$$
1+\beta\left(\theta_{1}, \theta_{2}\right)=\frac{2}{1-Q^{2}} g_{Q}\left(\frac{\theta_{1}}{2}-\frac{\theta_{2}}{2}-\frac{\pi}{2}, \frac{\theta_{1}}{2}+\frac{\theta_{2}}{2}-\frac{\pi}{2}\right) .
$$

Finally, recall $\beta_{n}=\beta\left(n \theta_{1}, n \theta_{2}\right)$ as we are calculating $1-(-1)^{n} \beta_{n}$. Then for $n$ even, we can use (107) to get

$$
\begin{aligned}
1-(-1)^{n} \beta_{n} & =\frac{2}{1-Q^{2}} g_{Q}\left(\frac{n \theta_{1}}{2}-\frac{n \theta_{2}}{2}, \frac{n \theta_{1}}{2}+\frac{n \theta_{2}}{2}\right) \\
& =\frac{2}{1-Q^{2}} g_{Q}\left(\frac{n \theta_{1}}{2}-\frac{n \theta_{2}}{2}-\frac{n \pi}{2}, \frac{n \theta_{1}}{2}+\frac{n \theta_{2}}{2}-\frac{n \pi}{2}\right) \\
& =\frac{2}{1-Q^{2}} g_{Q}(n \phi, n \psi),
\end{aligned}
$$

where we have used (101) and the fact that $g_{Q}$ is even and periodic with period $\pi$. Similarly, for $n$ odd, (108) gives the same result, and Lemma 18 is proved. 
7. Resonances and small divisors. In order to obtain periodic solutions of a nonlinear problem by perturbation from a known solution of a linearized problem by bifurcation methods, a major step is to show that the linearized operator is invertible on the orthogonal complement of the known solution. For example, this implies that the bifurcation problem is amenable to a Lyapunov-Schmidt decomposition; cf. [7]. Assuming (89), (90) of Theorem 14, we look for further conditions on the period $\Theta=(\bar{\theta}, \underline{\theta})$ such that the operator $\mathcal{M}-\mathcal{I}: \Delta \rightarrow \Delta$ is nonresonant in the sense that it is invertible on the complement of the solution $V_{a} \in \Delta$. That is, in light of (70), (67), we seek parameter values $\Theta$ such that

$$
\lambda_{1}^{ \pm}=-1 \quad \text { and } \quad \lambda_{n}^{ \pm} \neq(-1)^{n}
$$

for each $n \geq 2$, where $\lambda_{n}^{ \pm}$are given by (96). The equality here is a solvability condition for the linearized problem, while the inequalities represent an incommensurability or nonresonance condition imposing restrictions on allowable values of $\bar{\theta}$ and $\underline{\theta}$.

From (98), for finite $J>1$ we have

$$
\left|\beta_{n}\right| \leq 1+\frac{J^{2}+1}{2 J}=\frac{(J+1)^{2}}{2 J},
$$

so that, since $\beta_{n}$ is bounded, we can bound $\lambda_{n}^{ \pm}-(-1)^{n}$ away from zero by bounding $\beta_{n}$ away from $(-1)^{n}$. Thus the parameters $\underline{\theta}, \bar{\theta}$, and $J$, for which (75) holds, are nonresonant provided that

$$
\beta_{n} \neq(-1)^{n} \quad \text { for each } n \geq 2,
$$

where $\beta_{n}$ is given by $(98)^{4}$.

In light of (101), the nonresonance condition (109) is recast in terms of the parameters $\phi, \psi$, and $Q$ by the requirement that $g_{Q}(\phi, \psi)=0$, together with the condition that $g_{Q}(n \phi, n \psi) \neq 0$ for all $n \geq 2$. In fact, we have the following lower bound.

Lemma 19. For each $n \geq 2$, we have

$$
\left|\lambda_{n}^{ \pm}-(-1)^{n}\right| \geq \sqrt{\left|g_{Q}(n \phi, n \psi)\right|} .
$$

Proof. First, we use (103) in (110) to get

$$
\left|\beta_{n}\right|+1 \leq \frac{(J+1)^{2}}{2 J}+1=\frac{2}{1-Q^{2}}+1=\frac{3-Q^{2}}{1-Q^{2}},
$$

and now, referring to (70) and Lemmas 17 and 18, we get

$$
\begin{aligned}
\left|\lambda_{n}^{ \pm}-(-1)^{n}\right| & \geq \frac{\sqrt{\left|1-(-1)^{n} \beta_{n}\right|}}{\sqrt{1+\left|\beta_{n}\right|}} \\
& \geq \sqrt{\frac{2}{1-Q^{2}} g_{Q}(n \phi, n \psi)} \sqrt{\frac{1-Q^{2}}{3-Q^{2}}} \\
& \geq \sqrt{\left|g_{Q}(n \phi, n \psi)\right|},
\end{aligned}
$$

\footnotetext{
${ }^{4}$ Geometrically, the parameters will be resonant if the angles between states $q$ as drawn in Figures 7 and 8 are rational multiples of $\bar{\theta}$ and $\underline{\theta} \bmod \pi$, respectively. In this case, the matrix $M_{n}$ would have an eigenvalue of \pm 1 and we would not expect the $n$th mode to perturb to a solution of the nonlinear problem.
} 
since $0<Q<1$ implies $3-Q^{2} \leq 2$.

In order for the solution to be periodic at the linear level, we require that $\lambda_{1}^{ \pm}=-1$, or, equivalently,

$$
g_{Q}(\phi, \psi)=\sin ^{2} \psi-Q^{2} \sin ^{2} \phi=0,
$$

with

$$
0<\psi<\phi<\pi-\psi \quad \text { and } \quad 0<Q<1 .
$$

Note that this equation, which is equivalent to (75), can be regarded as fixing one parameter in terms of the other two.

The next lemma and its corollary, Theorem 21, state that the set of resonant parameters has measure zero.

Lemma 20. Almost every pair $(\phi, \psi)$ satisfying $0<\psi<\phi<\pi-\psi$ is nonresonant. That is, if we define $Q$ by (113), then the set

$$
\mathcal{H}=\left\{(\phi, \psi): \exists n>1 \text { s.t. } g_{Q}(n \phi, n \psi)=0\right\}
$$

has Lebesgue measure zero.

As a direct corollary we have the following theorem.

THEOREM 21. Let

$$
E \equiv\{\Theta=(\bar{\theta}, \underline{\theta}): \bar{\theta}, \underline{\theta}>0,0<\bar{\theta}+\underline{\theta}<\pi\} .
$$

Then there exists a set of full measure $E^{*} \subset E$ such that if $\Theta \in E^{*}$, then $\Theta$ is nonresonant in the sense that if $J$ is given in terms of $\Theta$ by (75), then the eigenvalues $\lambda_{n}^{ \pm}-(-1)^{n}$ of the linearized operator $\mathcal{M}-I$ are nonzero for all $n \geq 2$.

Proof of Lemma 20. To start, eliminate $Q$ from (112) to get

$$
Q \equiv Q(\phi, \psi)=\frac{\sin \psi}{\sin \phi},
$$

and plug this into $g_{Q}$ to get, for $n>1$,

$$
\begin{aligned}
g_{Q}(n \phi, n \psi) & =\sin ^{2}(n \psi)-\left(\frac{\sin \psi}{\sin \phi}\right)^{2} \sin ^{2}(n \phi) \\
& =\sin ^{2} \psi\left(\frac{\sin ^{2}(n \psi)}{\sin ^{2} \psi}-\frac{\sin ^{2}(n \phi)}{\sin ^{2} \phi}\right) .
\end{aligned}
$$

Now recall that the Chebyshev polynomials of the second kind are defined by

$$
S_{m}(x)=\frac{\sin (m+1) \theta}{\sin \theta}, \text { where } \quad x=\cos \theta,
$$

these being polynomials of degree $m$ with (distinct) roots in the unit interval. Thus defining $x_{\phi}=\cos \phi$ and $x_{\psi}=\cos \psi$, we have, for $n>1$,

$$
g_{Q}(n \phi, n \psi)=\sin ^{2} \psi\left(S_{n-1}^{2}\left(x_{\psi}\right)-S_{n-1}^{2}\left(x_{\phi}\right)\right) .
$$

We conclude that the pair $(\phi, \psi)$ is resonant if and only if

$$
S_{n-1}^{2}\left(x_{\psi}\right)=S_{n-1}^{2}\left(x_{\phi}\right) \quad \text { for some } \quad n>1 .
$$


Now for any fixed $\phi$ and $m$, there are at most $2 m$ values of $x$ such that $S_{m}^{2}(x)$ takes on the value $S_{m}^{2}\left(x_{\phi}\right)$, i.e., at most $2 m$ angles $\psi$ such that $g_{Q}(m \phi, m \psi)$ vanishes. Varying $m$, there are countably many angles $\psi$ that are resonant with $\phi$. Thus for fixed $\phi$, there is a countable (and thus measure zero) set of $\psi$ resonant with it. Now, if $\mathcal{H}$ is the resonant set and $\chi$ its characteristic function, then, by Fubini's theorem,

$$
\begin{aligned}
\mu(\mathcal{H}) & =\int_{0}^{\pi / 2} \int_{\psi}^{\pi-\psi} \chi d \phi d \psi \\
& =\int_{0}^{\pi / 2} \int_{0}^{\phi} \chi d \psi d \phi+\int_{\pi / 2}^{\pi} \int_{0}^{\pi-\phi} \chi d \psi d \phi \\
& =0 .
\end{aligned}
$$

This directly implies Lemma 20 and Theorem 21.

We now impose a further symmetry and, under this restriction, obtain explicit bounds for $g_{Q}(n \phi, n \psi)$. Since $0<\psi<\phi<\pi-\psi$, we get the largest range of $\psi$ by taking $\phi \equiv \pi / 2$. According to (101), this corresponds to taking $\bar{\theta}=\underline{\theta}$, so the length of the evolutions at the different entropy levels are the same. The following theorem gives algebraic decay rates for the small divisors in the symmetric case $\phi \equiv \pi / 2, \bar{\theta}=\underline{\theta}$.

THEOREM 22. Assume that

$$
\phi=\pi / 2, \quad \bar{\theta}=\underline{\theta} .
$$

Then for almost every $\psi \in(0, \pi / 2)$, there is a positive constant $C^{\prime}$ and exponent $r \geq 1$ such that the eigenvalues satisfy the estimate

$$
\left|\lambda_{n}^{ \pm}-(-1)^{n}\right| \geq \frac{C^{\prime}}{n^{r}}
$$

for all $n \geq 2$. In particular, if $\psi / \pi$ is the irrational root of a quadratic equation, we can take $r=1$.

Proof. Using assumption (116), (113) implies

$$
Q=\sin \psi
$$

To verify (117) we need to bound the function

$$
G_{n}(\psi) \equiv g_{Q}\left(n \frac{\pi}{2}, n \psi\right)=\sin ^{2}(n \psi)-\sin ^{2} \psi \sin ^{2}\left(n \frac{\pi}{2}\right) .
$$

Now for $n$ even, say $n=2 k$, we have

$$
G_{2 k}(\psi)=\sin ^{2}(2 k \psi),
$$

and for $n$ odd,

$$
G_{2 k+1}(\psi)=\sin ^{2}((2 k+1) \psi)-\sin ^{2} \psi .
$$

We can simplify these by trigonometric identities by writing

$$
\begin{aligned}
G_{2 k+1}(\psi) & =(\sin (2 k \psi) \cos \psi+\cos (2 k \psi) \sin \psi)^{2}-\sin ^{2} \psi \\
& =\sin ^{2}(2 k \psi)\left(\cos ^{2} \psi-\sin ^{2} \psi\right)+2 \sin (2 k \psi) \cos (2 k \psi) \sin \psi \cos \psi \\
& =\sin (2 k \psi)(\sin (2 k \psi) \cos (2 \psi)+\cos (2 k \psi) \sin (2 \psi)) \\
& =\sin (2 k \psi) \sin (2(k+1) \psi)
\end{aligned}
$$


Thus, if we can find some $\psi \in(0, \pi / 2)$, exponent $r \geq 1$, and positive constant $C>0$ such that

$$
|\sin (n \psi)|>\frac{C}{n^{r}}
$$

for each even $n>1$, then for $n$ odd we will also have

$$
\left|G_{n}(\psi)\right|=|\sin (n-1) \psi \sin (n+1) \psi|>\frac{C^{2}}{(n-1)^{r}(n+1)^{r}}>\frac{C^{2}}{n^{2 r}} .
$$

It follows from (111) that, for such $\psi$ and each $n>1$,

$$
\left|\lambda_{n}^{ \pm}-(-1)^{n}\right| \geq \sqrt{\left|G_{n}(\psi)\right|}>\frac{C}{n^{r}} .
$$

Thus it remains only to determine the angles $\psi$ such that the lower bound (120) holds. To this end, note that for $x \in[-\pi / 2, \pi / 2]$, we have

$$
|\sin x| \geq \frac{2|x|}{\pi}
$$

and thus also, if $x-m \pi \in[-\pi / 2, \pi / 2]$, then

$$
|\sin x|=|\sin (x-m \pi)| \geq \frac{2|x-m \pi|}{\pi} \geq 2 \operatorname{dist}(x / \pi, \mathbb{Z}) .
$$

Recall that a Liouville number is a number $\xi \in \mathbb{R}$ which is close to a rational, in the sense that for any integer $r$ we can find a rational $p / q$ such that

$$
\left|\xi-\frac{p}{q}\right|<\frac{1}{q^{r}}
$$

see, e.g., [13]. These $\xi$ form a set of measure zero, and in particular, Liouville's theorem states that if $\xi$ is the irrational root of a rational polynomial of degree $r^{\prime}$, then there is some constant $C>0$ such that

$$
\left|\xi-\frac{p}{q}\right|>\frac{C}{q^{r^{\prime}}}
$$

for all rationals $p / q$. In particular, if $\xi$ is the irrational root of a quadratic equation, we can take $r^{\prime}=2$.

Now, if $\xi=\psi / \pi$ is not a Liouville number, then there are $C=C(\psi)>0, r^{\prime} \geq 2$ such that (122) holds for all rationals $p / q$, and, in particular, taking $q=n$, we get

$$
\left|\frac{n \psi}{\pi}-p\right| \geq \frac{C}{n^{r^{\prime}-1}}
$$

for all integers $p$, so that

$$
\operatorname{dist}\left(\frac{n \psi}{\pi}, \mathbb{Z}\right) \geq \frac{C}{n^{r^{\prime}-1}}
$$

Combining this with (121), (120) follows and the proof of Theorem 22 is complete. 
In the symmetric case $\phi=\frac{\pi}{2}$ or $\underline{\theta}=\bar{\theta}$, it follows from (118), (119) that the solution is resonant if and only if $\psi / \pi \in \mathbb{Q}$, or, equivalently, $\bar{\theta} / \pi \in \mathbb{Q}$. We relate this observation to the effective wavespeed of solutions in the following theorem.

THEOREM 23. Characteristics return if and only if the group velocity, or speed of wave crests, is rational. Moreover, in the symmetric case $\bar{\theta}=\underline{\theta}$, the linearized solution is resonant if and only if the period is commensurate with the sound speed, i.e., if the characteristics return.

Proof. In the nondimensional system, the characteristic (phase) velocity and effective (group) velocity are given by

$$
c_{p}=1 \quad \text { and } \quad c_{g}=\frac{\bar{\theta}+\underline{\theta}}{\pi},
$$

respectively, (44). Thus, the (continued) forward characteristic through the point $\left(y_{0}, t_{0}\right)$ is the line $\left(y, t_{0}+y-y_{0}\right)$. Since the solution is periodic with period $2(\bar{\theta}+\underline{\theta})$ in space and $2 \pi$ in time, this characteristic returns if and only if

$$
\left(y, t_{0}+y-y_{0}\right)=\left(y_{0}, t_{0}\right)+(2 p(\bar{\theta}+\underline{\theta}), 2 q \pi)
$$

for some integers $p$ and $q$. This holds if and only if

$$
y-y_{0}=2 p(\bar{\theta}+\underline{\theta})=2 q \pi,
$$

or, using (123), if and only if $c_{g} \in \mathbb{Q}$. By symmetry, the backward characteristics satisfy the same conditions. Also note that one characteristic returns if and only if all characteristics do so.

In the symmetric case $\bar{\theta}=\underline{\theta} \equiv \theta$, if $\theta$ is a rational multiple of $\pi$, then say

$$
\theta=\frac{p}{q} \frac{\pi}{2} \quad \text { so that } \quad \psi=\frac{q-p}{q} \frac{\pi}{2} .
$$

According to (118) and (119), we get

$$
G_{2 q}(\psi)=G_{2 q+1}(\psi)=G_{2 q-1}(\psi)=0,
$$

so that these modes are resonant. It is interesting to note that these resonances always occur in triples of consecutive modes. Indeed, even if $q$ is odd, it is not the $q$ th mode that is resonant, but the $2 q$ th mode. Conversely, if $\theta$ is irrationally related to $\pi$, then $G_{k}(\psi) \neq 0$ for all $k>1$, and the solution is nonresonant.

Referring back to Figure 2, we see that the characteristic pattern as drawn is resonant, because the $\max /$ min characteristics return after four space periods, corresponding to the special parameter values

$$
\bar{\theta}=\underline{\theta}=\pi / 4 \text {. }
$$

On the other hand, the characteristic diagram shown in Figure 9 is nonresonant, and we see that characteristics move ergodically around a period, with no characteristic returning to itself.

Finally, we note that in the nonsymmetric case, we have

$$
\psi=\frac{\pi}{2}\left(1-c_{g}\right),
$$


and it is quite possible to have $c_{g} \in \mathbb{Q}$ and still be nonresonant, provided that $\phi$ is an irrational multiple of $\pi$. For example, we could take $\psi=\pi / 3$, so $c_{g}=1 / 3$. It follows that

$$
\frac{\sin (n \psi)}{\sin \psi}=0, \quad 1, \quad \text { or } \quad-1
$$

for each $n$, and by (115), our nonresonance condition becomes

$$
S_{n-1}\left(x_{\phi}\right) \neq 0 \quad \text { or } \quad \pm 1
$$

or, equivalently,

$$
\sin (n \phi) \neq \pm \sin \phi \quad \text { or } \quad 0 .
$$

Noting that $\sin (n \phi)=\operatorname{Im}\left\{e^{i n \phi}\right\}$, it follows that this nonresonance condition holds if $\phi$ is an irrational multiple of $\pi$.

7.1. A simulation of small divisors in the nonsymmetric case. In this subsection we address the small divisor issue in the nonsymmetric case with a numerical simulation. To start, the next lemma characterizes the zero set of $\beta_{n}(\underline{\theta}, \bar{\theta})-(-1)^{n}$ in the general (nonsymmetric) case.

LEMma 24. We have

$$
g_{Q}(\phi, \psi)=0 \quad \text { iff } \quad \psi= \pm h_{Q}(\phi),
$$

where

$$
h_{Q}(\phi)=\arcsin (Q \sin (\phi)) .
$$

Proof. This follows directly from (104).

It is clear from (104) that for each for $0<Q<1, g_{Q}$ is smooth, even, and periodic with period $\pi$ in both $\phi$ and $\psi$, while by (125), $h_{Q}$ is smooth, odd, $2 \pi$ periodic, and

$$
g_{Q}\left(\phi, h_{Q}(\phi)\right)=0
$$

for all $\phi$. Thus in these coordinates, the parameters $\phi$ and $Q$ are nonresonant provided that, for each $n \geq 2$, we have

$$
g_{Q}(n \phi, n \psi) \neq 0, \quad \text { where } \quad \psi=h_{Q}(\phi) .
$$

Now since $g_{Q}$ has period $\pi$ in $\phi$ and $\psi$, let $\Theta=(\phi, \psi) \in \mathbb{T}$, where

$$
\mathbb{T}=S^{1} \times S^{1}
$$

is two copies of the circle $S^{1}$ of radius $\pi / 2$,

$$
S^{1}=\left\{\theta: \theta \in\left[-\frac{\pi}{2}, \frac{\pi}{2}\right) \bmod \pi\right\},
$$

and define

$$
g(\Theta, Q)=g_{Q}(\Theta)
$$

so that

$$
g: \mathbb{T} \times(0,1) \rightarrow[0,1] .
$$


For the numerical simulation, let $A_{0}(Q)$ denote the zero set of $g$ at fixed $Q$, so that

$$
A_{0}(Q)=\{\Theta \in \mathbb{T}: g(\Theta, Q)=0\}
$$

so that by (124)

$$
A_{0}(Q)=\left\{\Theta=(\phi, \psi): \psi= \pm h_{Q}(\phi)\right\} .
$$

We now identify the salient properties of the zero sets $A_{0}(Q)$. Differentiating $h_{Q}(\phi)=\arcsin (Q \sin \phi)$ gives

$$
\begin{aligned}
h_{Q}^{\prime}(\phi) & =\frac{Q \cos \phi}{\sqrt{1-Q^{2} \sin ^{2} \phi}}, \\
h_{Q}^{\prime \prime}(\phi) & =-\frac{Q\left(1-Q^{2}\right) \sin \phi}{\sqrt{1-Q^{2} \sin ^{2} \phi}}, \quad \text { and } \\
\frac{\partial}{\partial Q} h_{Q}(\phi) & =\frac{\sin \phi}{\sqrt{1-Q^{2} \sin ^{2} \phi}} .
\end{aligned}
$$

Thus the function $\psi=h_{Q}(\phi)$ is an odd, increasing function for $-\frac{\pi}{2}<\phi<\frac{\pi}{2}$, and takes the values $\psi= \pm \arcsin Q$ at $\phi= \pm \frac{\pi}{2}$; and for each $Q \in(0,1), h_{Q}$ takes $\phi>0$ to $\psi>0$ and $\phi<0$ to $\psi<0$, while $-h_{Q}$ takes $\phi>0$ to $\psi<0$ and $\phi<0$ to $\psi>0$. It follows from $(126)$ that $h_{Q}(\phi)=\arcsin (Q \sin \phi)$ continues smoothly to the curve $h_{Q}(\phi)=-\arcsin (Q \sin \phi)$ at $\phi= \pm \pi / 2$. We record the salient properties of $A_{0}(Q)$ in the following lemma.

Lemma 25. The zero set $A_{0}(Q)$ in $\mathbb{T}$ consists of the union of two smooth curves

$$
A_{0}(Q)=\left(\phi, h_{Q}(\phi)\right) \cup\left(\phi,-h_{Q}(\phi)\right), \quad-\pi / 2 \leq \phi<\pi / 2,
$$

which is symmetric about the origin, and intersects itself only at the unique point $(0,0)$. Moreover, the curve $A_{0}(Q)$ is monotone in $Q$, and monotone and convex in $\phi$, in each quadrant of $\mathbb{T}$; and the length $L(Q)$ of the curve $A_{0}(Q)$ satisfies the uniform bound

$$
L(Q) \leq 2 \pi+4 \arcsin Q \leq 4 \pi .
$$

Proof. These properties follow directly from (125) and (126). In particular, for the estimate (127), use

$$
\begin{aligned}
L(Q) & \leq 4 \int_{0}^{\pi / 2} \sqrt{1+\left|h_{Q}^{\prime}(\phi)\right|^{2}} d \phi \leq 4 \int_{0}^{\pi / 2}\left(1+h_{Q}^{\prime}(\phi)\right) d \phi \\
& \leq 2 \pi+4 h_{Q}(\pi / 2)=2 \pi+4 \arcsin Q \leq 4 \pi .
\end{aligned}
$$

The curves $\psi= \pm h_{Q}(\phi)$ that define the resonance sets $A_{0}(Q)$ are drawn in Figure 10 for values of $Q$ ranging from $0<Q<1$. Figure 11 shows this zero set for the special parameter values $Q=3 / 4, \Theta=\left(\frac{\pi}{1+\sqrt{5}}, h_{\Theta}\left(\frac{\pi}{1+\sqrt{5}}\right)\right)$.

It follows directly from Theorem 21 that $\Theta$ is almost always nonresonant. For the purposes of this section, we record this in the following lemma.

LEMma 26. There exists a set of full measure $E_{1} \subset \mathbb{T}$,

$$
\mu\left\{E_{1}\right\}=\pi^{2} / 2
$$




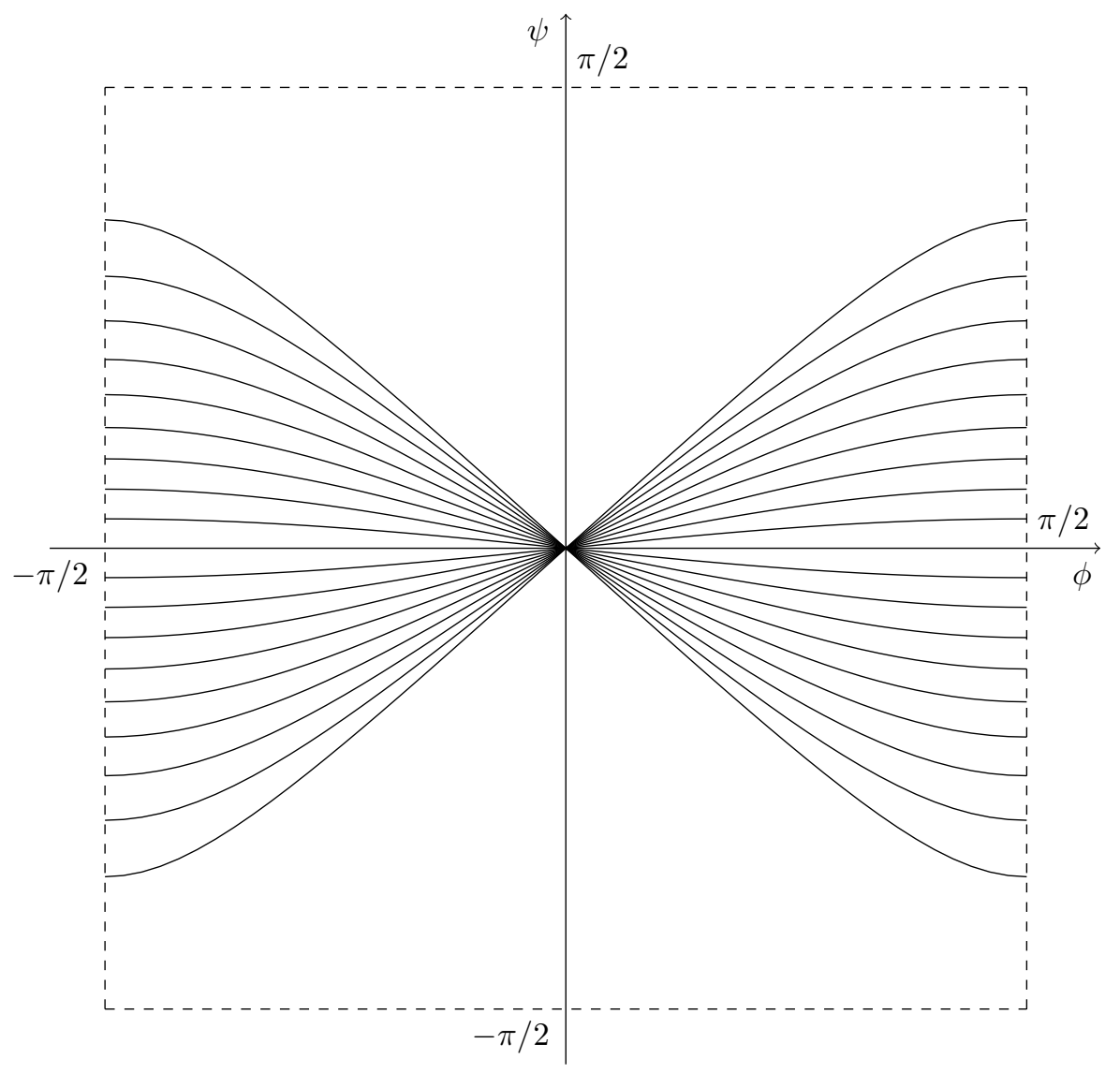

FIG. 10. The set $E_{0}$ consisting of the curves $\psi= \pm h_{Q}(\phi), 0<Q<1$.

such that if $\Theta \in E_{1}$, then $Q(\Theta) \in(0,1)$ is uniquely solvable by (112), and $\Theta$ is nonresonant in the sense that

$$
\begin{aligned}
g(\Theta, Q(\Theta)) & =0, \\
g(n \Theta, Q(\Theta)) & \neq 0, \quad n \geq 2 .
\end{aligned}
$$

Proof. By Lemma 25 we have that for $Q, Q^{\prime} \in(0,1), Q \neq Q^{\prime}$,

$$
A_{0}(Q) \cap A_{0}\left(Q^{\prime}\right)=\{(0,0)\} .
$$

It therefore follows that for each $\Theta \neq(0,0)$ there corresponds a unique value $Q(\Theta) \in$ $(0,1)$ such that

$$
\Theta=\left(\phi,-h_{Q}(\phi)\right) \quad \text { or } \quad \Theta=\left(\phi, h_{Q}(\phi)\right) .
$$

Solving for $Q$ in the expression $g_{Q}(\phi, \psi)=\sin ^{2} \psi-Q^{2} \sin ^{2} \phi=0$ gives

$$
Q(\Theta)=\sqrt{\frac{\sin ^{2} \psi}{\sin ^{2} \phi}}=\left|\frac{\sin \psi}{\sin \phi}\right|
$$




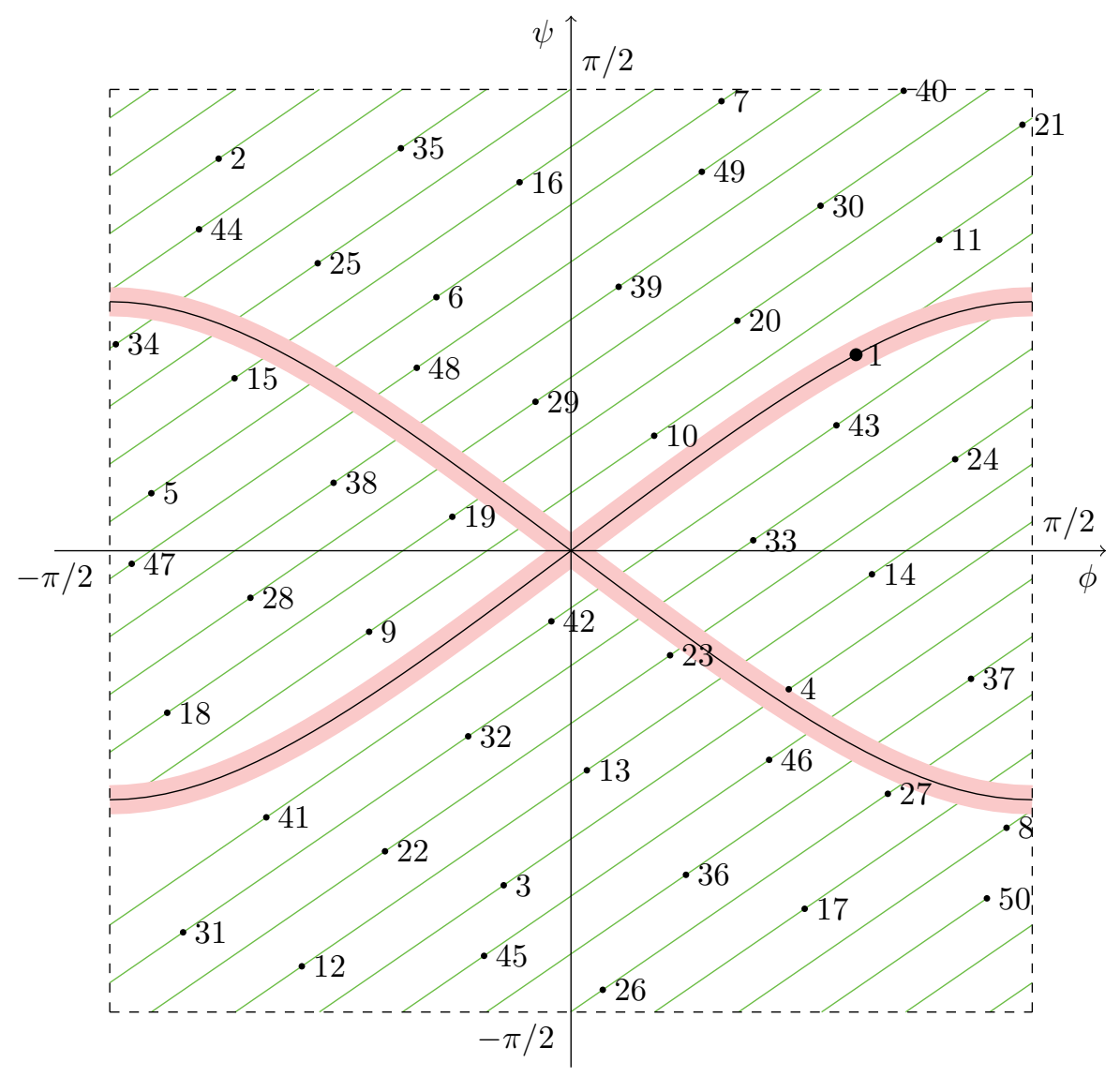

FIG. 11. The first $n \leq 50$ iterates $n \Theta$ avoiding $A_{\delta}(Q)$, for $Q=3 / 4$ and $\Theta=\left(\frac{\pi}{1+\sqrt{5}}, h_{Q}\left(\frac{\pi}{1+\sqrt{5}}\right)\right)$.

for $\phi \neq 0$, where we take the positive square root to get $Q>0$. Since $Q<1$, we require $0<|\sin \psi|<|\sin \phi|$. Thus the full measure of $E_{1}$ follows directly from Theorem 21, and (128) then follows from

$$
\mu\left\{E_{1}\right\}=\mu\{\Theta=(\phi, \psi) \in \mathbb{T}: 0<|\psi|<|\phi|\}=\pi^{2} / 2 .
$$

To get a sense of the order of the small divisors for a typical nonresonant $\Theta$ in the nonsymmetric case $\bar{\theta} \neq \underline{\theta}$, we conclude with a discussion of Figure 11 which gives a numerical simulation of the first fifty iterates $n \Theta$ for the special nonresonant value $\Theta=\left(\frac{\pi}{1+\sqrt{5}}, h_{\Theta}\left(\frac{\pi}{1+\sqrt{5}}\right)\right), Q=3 / 4$. Nonresonance implies that the iterates avoid the zero eigenvalue set $A_{0}(\Theta)$ drawn as the four symmetric curves through the origin in the figure.

The shaded region around $A_{0}(\Theta)$ is a neighborhood around $A_{0}(\Theta)$ such that the $n$th eigenvalue $\lambda_{n}$ will be bounded by $\epsilon \approx 0.05$ from zero when the $n$th iterate $n \Theta$ lies outside the shaded region. For these parameters, we have

$$
\min \left\{\left|\lambda_{n}\right|: 1<n \leq 50\right\}=\left|\lambda_{4}\right| \approx 0.5019 .
$$

Thus, for example, the plot in Figure 11 shows that the eigenvalues satisfy the bound in the first fifty modes. 
8. Conclusion. Our paper addresses the fundamental issue of why space-periodic solutions of the full $3 \times 3$ nonlinear compressible Euler equations do not decay by shock wave dissipation to the constant state average in each period. This is in contrast to the celebrated work of Glimm and Lax for $2 \times 2$ systems [6], in which all space-periodic solutions decay like $1 / t$. The present work addresses, quantitatively, issues raised by Shefter and Rosales [22] and Vaynblat [28], in numerical simulations that indicate the existence of some mechanism which allows for nondecaying solutions, and thus allows for dissipation free transmission of sound waves.

In our previous paper [26], we described the simplest physical mechanism by which shock formation and subsequent decay of solutions can be prevented. Simply stated, the presence of a varying entropy field leads to multiple reflections of simple waves, which can be aligned in a wave pattern in which compression and rarefaction are balanced along characteristics. This means that before any compression can form a shock, multiple interaction effects cause the wave to become rarefactive.

In this paper we derive closed form expressions for linearized solutions that realize the simplest possible wave pattern, identified in [26], that formally balances compression and rarefaction along characteristics. The results thus lend support to the claim that this wave pattern is also physically realized in nearby exact solutions of the fully nonlinear equations. The claim is further supported by the demonstration that for almost every period, the linearized operator (defined by the eigenvalue problem that expresses periodicity) is nonresonant in the sense that it is invertible on the complement of a one-dimensional kernel, with algebraic bounds on eigenvalues in certain special symmetric cases.

These results establish a framework for a new small divisor problem in bifurcation theory which, even without a complete mathematical proof, argue strongly for the existence of nearby time-periodic nonlinear solutions that have the same wave structure entailed by the linearized solutions. For example, the bifurcation problem is of quasilinear type, and so is beyond the direct application of known results, but similar problems have been resolved in the semilinear setting with the same estimates on the divisors, and weaker results on the structure of the kernel; cf. [2]. Moreover, our estimates on the spectrum of the linearized operator imply the bifurcation problem is amenable to a Lyapunov-Schmidt decomposition, an important first step in the resolution of the general problem [7]. ${ }^{5}$

In addition, these linearized time-periodic solutions have reasons to be interesting in their own right, not the least of which is the discovery of new phenomena when the sound speed is incommensurate with the period. That is, the wave crests propagate at the speed of the period (group velocity), not the sound speed (phase velocity), and these are irrationally related. The consistency of this possibility at the linearized level comes naturally out of the analysis, and indicates chaotic motion of sound wave trajectories relative to the solution periods. This then identifies a new ergodic way in which nearby nonlinear solutions can find a balance between compression and rarefaction along characteristics. Interestingly, we then discover that the operator is nonresonant only in case the wave speed is incommensurate with the period. Thus, for example, if the resonances are not just anomalies of the linearized problem, it suggests that this incommensurability is required for perturbation to nonlinear solutions.

Finally, we make some comments regarding the physical significance of these so-

\footnotetext{
${ }^{5}$ The authors develop the Lyapunov-Schmidt reduction and its implications to this bifurcation problem in detail in a forthcoming paper [27], and its full resolution is the topic of our ongoing research.
} 
lutions. First, the importance of solutions of such special structure to actual sound wave propagation in nature, and second the issue of the stability of these waves. Our view is that these new time-periodic structures are fundamental because they display, in the simplest consistent example, the mechanism by which dissipation free sound wave transmission is possible in the compressible Euler equations. Thus they anchor a paradigm for a much more general phenomenon, that of balancing compression and rarefaction along characteristics. We expect the analysis can be extended tremendously (for example, to arbitrary numbers of entropy jumps, and possibly the limit to smooth entropy profiles) and that it might eventually apply even to random entropy fields, which may be more relevant to actual physical sound wave propagation.

Regarding the issue of stability, our view is that it is the phenomenon (of balancing compression and rarefaction along characteristics) that is stable, rather than the very restrictive class of solutions considered here. That is, under a space-periodic perturbation from a periodic solution, the balance of compression and rarefaction along characteristics will be broken, and the solution will evolve until a new balance is established. Before this balance is achieved, some shock waves may form, causing the entropy profile to evolve in time. It is our contention that the nonlinear waves and entropy profile will continue to evolve in time, and evolution will proceed until a new balance of compression and rarefaction along characteristics is established. We expect this could be realized in some periodic or quasiperiodic fashion, and that for nonconstant entropy profiles, it will be an extremely rare event that solutions will decay to a constant state average in each period, as happens in the isentropic case. Thus the simplest way compression and rarefaction can be balanced along characteristics is of fundamental interest, independent of the special nature of solutions or stability considerations. In fact, the connections between unstable solutions, period-doubling, and chaos in bifurcation theory lead us to believe that unstable solutions in this setting could well provide a handle on new and even more interesting chaotic evolution in the compressible Euler equations. Our view is that these new linearized solutions open the door to interesting new conjectures, and provide intuition and a starting point for the discovery of new phenomena.

\section{REFERENCES}

[1] S. Alinhac and P. GÉrard, Pseudo-differential operators and the Nash-Moser theorem, Grad. Stud. Math. 82, American Mathematical Society, Providence, RI, 2007.

[2] W. Craig and C. E. Wayne, Newton's method and periodic solutions of nonlinear wave equations, Comm. Pure Appl. Math., 46 (1993), pp. 1409-1498.

[3] C. M. Dafermos, Hyperbolic Conservation Laws in Continuum Physics, Springer-Verlag, Berlin, 2000.

[4] K. Deimling, Nonlinear Functional Analysis, Springer-Verlag, Berlin, 1985.

[5] J. GLimm, Solutions in the large for nonlinear hyperbolic systems of equations, Comm. Pure Appl. Math., 18 (1965), pp. 697-715.

[6] J. Glimm And P. D. Lax, Decay of Solutions of Systems of Nonlinear Hyperbolic Conservation Laws, Memoirs Amer. Math. Soc. 101, American Mathematical Society, Providence, RI, 1970.

[7] M. Golubitsky and D. G. Schaeffer, Singularities and Groups in Bifurcation Theory, Appl. Math. Sci. 51, Springer-Verlag, New York, 1985.

[8] J. M. Greenberg, Smooth and time-periodic solutions to the quasilinear wave equation, Arch. Rational Mech. Anal., 60 (1975), pp. 29-50.

[9] J. M. Greenberg and M. Rascle, Time-periodic solutions to systems of conservation laws, Arch. Rational Mech. Anal., 115 (1991), pp. 395-407.

[10] F. John, Formation of singularities in one-dimensional nonlinear wave opagation, Comm. Pure Appl. Math., 27 (1974), pp. 377-405. 
[11] P. D. Lax, Hyperbolic systems of conservation laws, II, Comm. Pure Appl. Math., 10 (1957), pp. 537-566.

[12] P. D. Lax, Development of singularities of solutions of nonlinear hyperbolic partial differential equations, J. Mathematical Phys., 5 (1964), pp. 611-613.

[13] W. J. LeVeque, Fundamentals of Number Theory, Addison-Wesley, Reading, MA, 1977.

[14] T.-T. LI, D. SerRe, AND Z. HAO, The generalized Riemann problem for the motion of elastic strings, SIAM J. Math. Anal., 23 (1992), pp. 1189-1203.

[15] T.-P. LiU, Development of singularities in the nonlinear waves for quasilinear hyperbolic partial differential equations, J. Differential Equations, 33 (1979), pp. 92-111.

[16] T.-P. Liu, Quasilinear hyperbolic systems, Comm. Math. Phys., 68 (1979), pp. 141-172.

[17] A. Majda, Compressible Fluid Flow and Systems of Conservation Laws in Several Space Variables, Appl. Math. Sci. 53, Springer-Verlag, New York, 1984.

[18] A. Majda AND R. Rosales, Resonantly interacting weakly nonlinear hyperbolic waves. I. A single space variable, Stud. Appl. Math., 71 (1984), pp. 149-179.

[19] A. Majda, R. Rosales, And M. Schonbeck, A canonical system of integrodifferential equations arising in resonant nonlinear acoustics, Stud. Appl. Math., 79 (1988), pp. 205-262.

[20] R. L. Pego, Some explicit resonating waves in weakly nonlinear gas dynamics, Stud. Appl. Math., 79 (1988), pp. 263-270.

[21] P. H. Rabinowitz, Periodic solutions of nonlinear hyperbolic partial differential equations, Comm. Pure Appl. Math., 20 (1967), pp. 145-205.

[22] M. Shefter and R. Rosales, Quasiperiodic solutions in weakly nonlinear gas dynamics. I. Numerical results in the inviscid case, Stud. Appl. Math., 103 (1999), pp. 279-337.

[23] J. Smoller, Shock Waves and Reaction-Diffusion Equations, Springer-Verlag, New York, 1982.

[24] B. Temple And R. Young, The large time existence of periodic solutions for the compressible Euler equations, Mat. Contemp., 11 (1996), pp. 171-190.

[25] B. Temple And R. Young, The large time stability of sound waves, Comm. Math. Phys., 179 (1996), pp. 417-466.

[26] B. Temple and R. Young, A paradigm for time-periodic sound wave propagation in the compressible Euler equations, Methods Appl. Anal., 16 (2009), pp. 341-363.

[27] B. Temple And R. Young, A Liapunov-Schmidt reduction for time-periodic solutions of the compressible Euler equations, submitted. Available online at http://www.math .ntnu.no/conservation/2008/035.html.

[28] D. Vaynblat, The Strongly Attracting Character of Large Amplitude Nonlinear Resonant Acoustic Waves Without Shocks. A Numerical Study, Ph.D. thesis, MIT, Boston, MA, 1996.

[29] R. Young, Sup-norm stability for Glimm's scheme, Comm. Pure Appl. Math., 46 (1993), pp. 903-948.

[30] R. Young, Exact solutions to degenerate conservation laws, SIAM J. Math. Anal., 30 (1999), pp. $537-558$.

[31] R. Young, Periodic solutions for conservation laws, Contemp. Math. 255, American Mathematical Society, Providence, RI, 2000, pp. 239-256.

[32] R. Young, The p-system. II: The vacuum, in Evolution Equations (Warsaw 2001), R. Picard, M. Reissig, and W. Zajaczkowski, eds., Banach Center Publ. 60, Polish Acad. Sci., Warsaw, 2003, pp. 237-252.

[33] R. Young, Sustained solutions for conservation laws, Comm. Partial Differential Equations, 26 (2001), pp. 1-32.

[34] R. Young, Isentropic gas dynamics with large data, in Hyperbolic Problems: Theory, Numerics, Applications, T. Hou and E. Tadmor, eds., Springer, Berlin, 2003, pp. 929-939.

[35] E. ZEHNDER, Generalized implicit function theorems with applications to some small divisor problems, Comm. Pure Appl. Math., 28 (1975), pp. 91-140. 